\title{
Helmholtz Energy Transformations of Common Cubic Equations of State for Use with Pure Fluids and Mixtures*
}

\author{
Ian H. Bell ${ }^{1}$ and Andreas Jäger ${ }^{2}$ \\ ${ }^{1}$ National Institute of Standards and Technology, \\ Boulder, CO 80305 \\ ${ }^{2}$ Technische Universität Dresden, Professur für Technische Thermodynamik, Helmholtzstr. \\ 14, 01062 Dresden, Germany \\ ian.bell@nist.gov \\ andreas.jaeger@tu-dresden.de
}

\begin{abstract}
Comprehensive sets of derivatives of Helmholtz energy transformations of several common cubic equations of state are presented. These derivatives can be used for implementing cubic equations of state into complex multi-fluid mixture models when no multiparameter equation of state is available for a mixture component. Thus, pure fluids (or mixtures) for which no accurate model exists in literature can be modeled with a relatively small set of fluid property data. Composition derivatives have been calculated for the cases where the last mole fraction is either an independent or dependent variable. Analytic derivatives are presented up to fourth order in the independent variables combined with composition derivatives up to third order; this set covers the common requirements for derivatives needed in the state-of-the-art thermophysical property libraries. A C++ implementation of the presented analyses, data for computer code validation, and information about the computer algebra tools used to calculate the intermediate derivatives are provided as supplementary information.
\end{abstract}

Key words: cubic equations of state; derivatives; Helmholtz energy; mixture models; thermodynamic potential.

Accepted: April 14, 2016

Published: 2016

http://dx.doi.org/10.6028/jres.121.011

\section{Helmholtz-Energy-Explicit Models}

Thermodynamic properties of fluids can be calculated by means of different types of equations of state. This section describes those that are formulated in terms of the Helmholtz energy. They evolved from the virial equation of state and became common when pure fluids had been characterized over wide ranges of states and in multiple properties because all this information could be represented with high accuracy in a single formulation of the Helmholtz energy.

\subsection{Helmholtz Energy Potential}

Multiproperty formulations take advantage of the laws and axioms of thermodynamics which rank certain combinations of independent and dependent variables as fundamental relations because they contain

\footnotetext{
* Commercial equipment, instruments, or materials are identified only in order to adequately specify certain procedures. In no case does such identification imply recommendation or endorsement by the National Institute of Standards and Technology, nor does it imply that the products identified are necessarily the best available for the purpose.
} 
all thermodynamic information about a particular system. Such all-inclusiveness is a mark of potential functions, in this case of thermodynamic potentials. The best known of these are [1]

\begin{tabular}{cc}
\hline Dependent & Independent \\
\hline$u$ & $s, v$ \\
$h$ & $s, p$ \\
$a$ & $v, T$ \\
$g$ & $p, T$ \\
\hline
\end{tabular}

where $u$ is the internal energy, $h$ is the enthalpy, $s$ is the entropy, $a$ is the Helmholtz energy, $g$ is the Gibbs energy, $v$ is the volume, $T$ is the temperature, and $p$ is the pressure. Thermodynamic potentials are equivalent because they are Legendre-transforms of each other. The two potentials with measurable quantities as independent variables are the Gibbs energy $g$ and the Helmholtz energy $a$. The Gibbs energy in the form of the chemical potential is the basis of phase equilibrium calculations in chemical engineering while formulations of the Helmholtz energy have been preferably applied to correlate wide-ranging data and properties of pure fluids with high accuracy. Such formulations are implemented in the current state-ofthe-art thermophysical property libraries: NIST REFPROP [2], CoolProp [3], and TREND [4].

The Helmholtz energy $a$ is commonly expressed as a sum of the ideal-gas contribution $a^{0}$ and the residual contribution $a^{\mathrm{r}}$. In practice, the Helmholtz energy $a$ is non-dimensionalized by the product of the gas constant $R$ and the temperature $T$, and the independent variables are transformed as well, yielding

$$
\alpha(\tau, \delta)=\frac{a}{R T}=\alpha^{\mathrm{r}}(\tau, \delta)+\alpha^{0}(\tau, \delta)
$$

where the non-dimensionalized density is given by $\delta=\rho / \rho_{\mathrm{r}}$ and the reciprocal reduced temperature is given by $\tau=T_{\mathrm{r}} / T$. The reducing parameters $\rho_{\mathrm{r}}$ and $T_{\mathrm{r}}$ are usually equal to the critical values for pure fluids. The reducing models used for $\rho_{\mathrm{r}}$ and $T_{\mathrm{r}}$ when modeling mixtures are described further in Sec. 2.2.

The link between Helmholtz energy formulations and pressure-explicit equations of state is the derivative

$$
p=-\left(\frac{\partial a}{\partial v}\right)_{T}
$$

or in reduced form

$$
\frac{p}{\rho R T}=1+\delta\left(\frac{\partial \alpha^{\mathrm{r}}}{\partial \delta}\right)_{\tau}
$$

As an example, the enthalpy and entropy can be obtained from

$$
\begin{gathered}
\frac{h}{R T}=\tau\left[\left(\frac{\partial \alpha^{0}}{\partial \tau}\right)_{\delta}+\left(\frac{\partial \alpha^{\mathrm{r}}}{\partial \tau}\right)_{\delta}\right]+\delta\left(\frac{\partial \alpha^{\mathrm{r}}}{\partial \delta}\right)_{\tau}+1 \\
\frac{s}{R}=\tau\left[\left(\frac{\partial \alpha^{0}}{\partial \tau}\right)_{\delta}+\left(\frac{\partial \alpha^{\mathrm{r}}}{\partial \tau}\right)_{\delta}\right]-\alpha^{0}-\alpha^{\mathrm{r}} .
\end{gathered}
$$

Additional derivatives can be found in the book of Span [5] or the paper of Lemmon et al. [6].

In this work we consider only the residual Helmholtz energy $\alpha^{\mathrm{r}}$ and not the ideal-gas Helmholtz energy $\alpha^{0}$; the residual Helmholtz energy can be obtained from an explicit transformation of pressure-explicit cubic equations of state, while the integration of the ideal-gas specific heat is required to obtain $\alpha^{0}$. 


\subsection{Pure Fluids}

Helmholtz energy formulations are able to represent the experimental data available within their uncertainties but are relatively complex and thus difficult to evaluate. Furthermore, the multiparameter models are empirical in nature, and must be correlated to large datasets of high-accuracy experimental data to yield high-accuracy calculations of thermodynamic properties.

These multiparameter equations represent the state-of-the-art in high-accuracy representations of pure fluid properties. For instance, the residual part of the equation of state for propane of Lemmon et al. [7] has the form

$$
\alpha^{\mathrm{r}}=\sum_{k=1}^{5} N_{k} \delta^{d_{k}} \tau^{t_{k}}+\sum_{k=6}^{11} N_{k} \delta^{d_{k}} \tau^{t_{k}} \exp \left(-\delta^{l_{k}}\right)+\sum_{k=12}^{18} N_{k} \delta^{d_{k}} \tau^{t_{k}} \exp \left(-\eta_{k}\left(\delta-\varepsilon_{k}\right)^{2}-\beta_{k}\left(\tau-\gamma_{k}\right)^{2}\right),
$$

which is a combination of polynomial-like (the first summation), polynomial plus exponential (the second summation), and Gaussian terms (the third summation). All subscripted symbols represent adjustable parameters.

\subsection{Mixtures (the Multi-Fluid Mixture Model)}

For Helmholtz equations of state, the multi-fluid mixture model, see Refs. [8-14], is regarded as stateof-the-art. This model has the advantage that highly accurate formulations for pure fluids can be directly used in the mixture model; all pure fluid contributions to the reduced residual Helmholtz energy are evaluated at the same reduced temperature $\tau$ and reduced density $\delta$, not at the same temperature $T$ and density $\rho$. Mixture properties are then obtained by combining the reduced Helmholtz energies of the pure fluids. Hence, in order to apply this model, Helmholtz-energy-explicit equations of state must be available for all components in the mixtures.

In the multi-fluid models implemented in NIST REFPROP [2], CoolProp [3], and TREND [4], the contributions from the pure fluids are combined in the following manner to add the composition dependence

$$
\alpha^{\mathrm{r}}=\sum_{i} x_{i} \alpha_{\mathrm{oi}}^{\mathrm{r}}(\tau, \delta)+\sum_{i=1}^{N-1} \sum_{j=i+1}^{N} x_{i} x_{j} F_{i j} \alpha_{i j}^{\mathrm{r}}(\tau, \delta)
$$

where $\alpha_{\mathrm{o} i}^{\mathrm{r}}$ is the contribution from the $i$-th pure fluid, which is in general given by a multiparameter equation of state like that shown above in Sec. 1.2. The term $\alpha_{i j}^{\mathrm{r}}$ is a departure function used to shape the thermodynamic surface for a given binary pair, and $F_{i j}$ is an adjustable parameter that can be used to scale departure functions developed for common families of binary pairs. Equation (7) corresponds to the GERG mixture model [11,12].

According to the multi-fluid model, the reducing temperature $T_{\mathrm{r}}$ and the reducing density $\rho_{\mathrm{r}}$ are functions of composition $T_{\mathrm{r}}(\bar{x})$ and $\rho_{\mathrm{r}}(\bar{x})$ [11,12]. In the limiting case of pure fluids, the reducing temperature becomes the critical temperature of the pure fluid $T_{\mathrm{c}}$ and the reducing density becomes the critical density of the pure fluid $\rho_{\mathrm{c}}$.

Some mixture properties (like pressure or enthalpy) can be straightforwardly obtained from $\tau$ and $\delta$ partial derivatives of $\alpha$. Other properties, like fugacities or fugacity coefficients, require composition derivatives. For instance, the fugacity can be given by $[11,12]$

$$
f_{i}=x_{i} \rho R T \exp \left(\frac{\partial n \alpha^{\mathrm{r}}}{\partial n_{i}}\right)_{T, V, n_{j}} .
$$


Details of the derivations required to obtain the fugacity in terms of derivatives of $\alpha^{\mathrm{r}}$ are given in Refs. [11,12].

\section{Cubic Pressure-Explicit Equations of State}

While the development of multiparameter Helmholtz energy formulations requires experimental data of multiple properties of good quality over a broad temperature and pressure range, the only information needed to use cubic equations of state are the critical temperature, critical pressure, and acentric factor (or vapor pressure curves in the case of the Mathias-Copeman equation for $a_{i i}$ ). The most frequently used types of cubic equations of state are the equations by Soave, Redlich, and Kwong (SRK) (as in Soave [15] and Redlich and Kwong [16]) and Peng and Robinson (PR) [17].

As noted by Michelsen and Mollerup [18], cubic equations of state can be expressed in a common form given by

$$
p=\frac{R T}{v-b_{\mathrm{m}}}-\frac{a_{\mathrm{m}}(T)}{\left(v+\Delta_{1} b_{\mathrm{m}}\right)\left(v+\Delta_{2} b_{\mathrm{m}}\right)}
$$

where $v$ is the molar volume in $\mathrm{m}^{3} \cdot \mathrm{mol}^{-1}, R$ is the universal gas constant in $\mathrm{J} \cdot \mathrm{mol}^{-1} \cdot \mathrm{K}^{-1}, T$ is the temperature in $\mathrm{K}, a_{\mathrm{m}}$ is the mixture attractive term in $\mathrm{J} \cdot \mathrm{m}^{3} \cdot \mathrm{mol}^{-2}$, and $b_{\mathrm{m}}$ is the mixture covolume in $\mathrm{m}^{3} \cdot \mathrm{mol}^{-1}$. Other even more general functional forms have been proposed that cover an even wider range of potential structures for cubic and cubic-like equations of state. For instance, the reviews of Poling et al. [19], Wei and Sadus [20], and Valderramma [21] provide a thorough discussion of the multitude of cubiclike functional forms that have been developed since the equation of state of van der Waals in 1873 [22].

In this work, Helmholtz energy translations for cubic equations of state are presented. All cubic equations have been handled in a universal formulation. The presented formulations also allow for the use of cubic equations of state in multi-fluid models, i.e., the Helmholtz energy contributions for the pure fluid in multi-fluid models can be calculated either from multiparameter equations of state or cubic equations of state.

\subsection{Cubic Parameters}

For cubic equations of state, usually one-fluid mixture models are used, see e.g., Soave [15] or Peng and Robinson [17]. In the case of one-fluid mixture models, the mixture properties are not modeled by combining the pure fluid contributions (as in the multi-fluid mixture model) but by applying mixing rules to fluid specific parameters for a hypothetical pure fluid. For instance, quadratic mixing rules in terms of composition are usually applied to the attraction parameter $a_{\mathrm{m}}$, and linear mixing rules are applied to the covolume $b_{\mathrm{m}}$.

For all the investigated equations of state, the mixture parameters $a_{\mathrm{m}}$ and $b_{\mathrm{m}}$ are given by quadratic and linear mixing rules in molar composition, respectively. Thus the forms for $a_{\mathrm{m}}$ and $b_{\mathrm{m}}$ are given by

$$
\begin{gathered}
a_{\mathrm{m}}(T, \bar{x})=\sum_{i} \sum_{j} x_{i} x_{j} a_{i j}(T) \\
b_{\mathrm{m}}(\bar{x})=\sum_{i} x_{i} b_{i i}
\end{gathered}
$$

The cross attractive term $a_{i j}(T)$ is given by the form

$$
a_{i j}(T)=\left(1-k_{i j}\right) \sqrt{a_{i i}(T) a_{j j}(T)}
$$

where $k_{i j}=0$ if $i=j$. 
In the classical cubic formulations of Peng-Robinson [17] and Soave-Redlich-Kwong [15], the form of $a_{i i}$ was given by

$$
a_{i i}=a_{0, i i}\left[1+m_{i i}\left(1-\sqrt{\frac{T}{T_{\mathrm{c}, i}}}\right)\right]^{2}
$$

where $a_{0, i i}$ and $m_{i i}$ are fluid-specific constant terms particular to the equation of state. Table 1 gives the forms of the constants for three common cubic equations of state.

Table 1. Common implementations of the cubic equations of state ( $R$ : universal gas constant in $\mathrm{J} \cdot \mathrm{mol}^{-1} \cdot \mathrm{K}^{-1}, T_{\mathrm{C}, i}$ : critical temperature in $\mathrm{K}, p_{\mathrm{c}, i}:$ critical pressure in $\mathrm{Pa}, \omega_{i}:$ acentric factor of the pure component).

\begin{tabular}{|c|c|c|c|c|c|}
\hline Name & $a_{0, i i}$ & $b_{i i}$ & $m_{i i}$ & $\Delta_{1}$ & $\Delta_{2}$ \\
\hline van der Waals [22] & $0.42188 \frac{R^{2} T_{\mathrm{c}, i}{ }^{2}}{p_{\mathrm{c}, i}}$ & $0.125 \frac{R T_{\mathrm{c}, i}}{p_{\mathrm{c}, i}}$ & 0 & 0 & 0 \\
\hline Soave-Redlich-Kwong [15] & $0.42747 \frac{R^{2} T_{\mathrm{c}, i}{ }^{2}}{p_{\mathrm{c}, i}}$ & $0.08664 \frac{R T_{\mathrm{c}, i}}{p_{\mathrm{c}, i}}$ & $0.48+1.574 \omega_{i}-0.176 \omega_{i}^{2}$ & 1 & 0 \\
\hline Peng-Robinson [17, 23] & $0.45724 \frac{R^{2} T_{\mathrm{c}, i}{ }^{2}}{p_{\mathrm{c}, i}}$ & $0.07780 \frac{R T_{\mathrm{c}, i}}{p_{\mathrm{c}, i}}$ & $\dagger$ & $1+\sqrt{2}$ & $1-\sqrt{2}$ \\
\hline
\end{tabular}

In engineering practice, it has been found that more flexibility in the form of $a_{i i}$ is required, particularly to fit the vapor pressure curves of polar fluids. Numerous attempts have been made to arrive at an $a_{i i}$ term that gives additional flexibility, and one of the most commonly used forms is that of Mathias and Copeman [24]

$$
a_{i i}=a_{0, i i}\left[1+C_{1, i}\left(1-\sqrt{\frac{T}{T_{\mathrm{c}, i}}}\right)+C_{2, i}\left(1-\sqrt{\frac{T}{T_{\mathrm{c}, i}}}\right)^{2}+C_{3, i}\left(1-\sqrt{\frac{T}{T_{\mathrm{c}, i}}}\right)^{3}\right]^{2}
$$

where, in the absence of sufficient experimental data, $C_{1, i}$ can be set to $m_{i i}$ and $C_{2, i}$ and $C_{3, i}$ can be set to zero to yield a predictive scheme for $a_{i i}$. Therefore, Eq. (13) can be considered a special case of Eq. (14), and we can carry out the derivations through the use of Eq. (14) with no loss in generality. The MathiasCopeman equation is commonly used because it is straightforward to obtain the coefficients $C_{n, i}$, either by optimization, or from tabulated values (like those of Horstmann et al. [25] for more than 1000 fluids).

If the mixture has only one component (i.e., a pure fluid), the $a_{\mathrm{m}}$ and $b_{\mathrm{m}}$ terms simplify to

$$
\begin{gathered}
a_{\mathrm{m}}(T)=a_{11}(T) \\
b_{\mathrm{m}}=b_{11} .
\end{gathered}
$$

Other mixing rules have been proposed for the mixture covolume $b_{\mathrm{m}}$, including quadratic mixing rules (see for instance McFarlane et al. [26]). The extension of the derivatives of $b_{\mathrm{m}}$ to quadratic mixing rules is not demonstrated in this work but can easily be done if needed; linear mixing rules for $b_{\mathrm{m}}$ are more prevalent in the literature. 


\subsection{Reducing Values $\rho_{\mathrm{r}}$ and $T_{\mathrm{r}}$}

When translating cubic equations into the reduced residual Helmholtz energy, the reducing parameters $\rho_{\mathrm{r}}$ and $T_{\mathrm{r}}$ have to be handled depending on the way that the model is to be used.

\subsubsection{Pure Fluid and Multi-Fluid Mixture}

The first case is that cubic equations are used to model a pure fluid (either for pure fluid properties or as a contribution $\alpha^{\mathrm{r}}{ }_{\text {oi }}$ in a multi-fluid mixture). In this case, all temperatures $T$ in the cubic equation are replaced by $T_{\mathrm{r}} / \tau$ and all densities $\rho$ are replaced by $\rho_{\mathrm{r}} \cdot \delta$, with $T_{\mathrm{r}}=T_{\mathrm{c}}$ and $\rho_{\mathrm{r}}=\rho_{\mathrm{c}}$; the reducing parameters need to be equal to the critical parameters of the fluid. The inverse reduced temperature $\tau$ and the reduced density $\delta$ for models explicit in the non-dimensionalized Helmholtz energy is as described in Sec. 1.

\subsubsection{Mixture as One-Fluid}

The second case is that Helmholtz-energy-translated cubic equations of state are used in a one-fluid mixture model, which corresponds to the "usual way" of handling mixtures with cubic equations of state. In this case, all temperatures $T$ in the cubic equation are replaced by $T_{\mathrm{r}} / \tau$ and all densities are replaced by $\rho_{\mathrm{r}} \cdot \delta$, with $T_{\mathrm{r}}$ and $\rho_{\mathrm{r}}$ being parameters that can be arbitrarily chosen. In this work, $T_{\mathrm{r}}=1 \mathrm{~K}$ and $\rho_{\mathrm{r}}=1 \mathrm{~mol} \cdot \mathrm{m}^{-3}$.

\subsection{Molar Composition}

In general, a mixture of $N$ components is specified by its molar composition - though in some fields mass fractions are more prevalent. Additionally, in other literature, mole numbers are taken to be the independent variables rather than mole fractions. The mole fractions can be considered in two ways:

- all of the mole fractions $x_{1}$ to $x_{N}$ are treated as independent variables

- only the first $N-1$ mole fractions are considered as independent variables.

If $x_{N}$ is a dependent variable, it is therefore determined from the other mole fractions from

$$
x_{N}=1-\sum_{i=1}^{N-1} x_{i} .
$$

The distinction concerning $x_{N}$ only appears in the composition derivatives of the mixture terms $a_{\mathrm{m}}$ and $b_{\mathrm{m}}$. This dependency is implicitly invoked in all other equations and composition derivatives.

\subsection{Conversion to Residual Non-Dimensionalized Helmholtz Energy}

As described above pressure-explicit cubic equations of state can be transformed into a Helmholtzenergy-explicit equation of state with reciprocal reduced temperature and reduced density as independent variables. Thus in the first step, the necessary variable substitutions are made in Eq. (9) to yield

$$
p=\frac{T_{\mathrm{r}}}{\tau} \frac{R}{\frac{1}{\delta \rho_{\mathrm{r}}}-b_{\mathrm{m}}}-\frac{a_{\mathrm{m}}(\tau)}{\left(\frac{1}{\delta \rho_{\mathrm{r}}}+\Delta_{1} b_{\mathrm{m}}\right)\left(\frac{1}{\delta \rho_{\mathrm{r}}}+\Delta_{2} b_{\mathrm{m}}\right)}
$$

or

$$
p=\frac{T_{\mathrm{r}}}{\tau} \frac{\delta \rho_{\mathrm{r}} R}{1-b_{\mathrm{m}} \delta \rho_{\mathrm{r}}}-\frac{\delta^{2} \rho_{\mathrm{r}}^{2} a_{\mathrm{m}}(\tau)}{\left(1+\Delta_{1} b_{\mathrm{m}} \delta \rho_{\mathrm{r}}\right)\left(1+\Delta_{2} b_{\mathrm{m}} \delta \rho_{\mathrm{r}}\right)} .
$$


The compressibility factor $Z$ is given by

$$
Z=\frac{p}{\delta \rho_{\mathrm{r}} R T}
$$

and since

$$
Z=1+\delta\left(\frac{\partial \alpha^{\mathrm{r}}}{\partial \delta}\right)_{\tau}
$$

the derivative of the residual non-dimensional Helmholtz energy with respect to $\delta$ is given by

$$
\left(\frac{\partial \alpha^{\mathrm{r}}}{\partial \delta}\right)_{\tau}=\frac{Z-1}{\delta}=\frac{\frac{p}{\delta \rho_{\mathrm{r}} R T}-1}{\delta}
$$

Therefore, in substituting $p$ from Eq. (19) into the right-hand side of Eq. (22), we obtain

$$
\frac{Z-1}{\delta}=\frac{b_{\mathrm{m}} \rho_{\mathrm{r}}}{1-b_{\mathrm{m}} \delta \rho_{\mathrm{r}}}-\frac{\tau a_{\mathrm{m}}}{R T_{\mathrm{r}}} \frac{\rho_{\mathrm{r}}}{\left(1+\Delta_{1} b_{\mathrm{m}} \delta \rho_{\mathrm{r}}\right)\left(1+\Delta_{2} b_{\mathrm{m}} \delta \rho_{\mathrm{r}}\right)} .
$$

The residual non-dimensional Helmholtz energy is then obtained from

$$
\alpha^{\mathrm{r}}=\int_{0}^{\delta} \frac{Z-1}{\delta} \mathrm{d} \delta \text {. }
$$

The integral for $\alpha^{\mathrm{r}}$ can be separated into two pieces and expressed in the form

$$
\alpha^{\mathrm{r}}=\psi^{(-)}-\frac{\tau a_{\mathrm{m}}}{R T_{\mathrm{r}}} \psi^{(+)} .
$$

The $\psi^{(-)}$and $\psi^{(+)}$terms are functions of $\delta$ but not of $\tau$ and arise from integrating portions of $(Z-1) / \delta$, while the remaining term $\frac{\tau a_{\mathrm{m}}}{R T_{\mathrm{r}}}$ is a function of $\tau$ but not of $\delta$. by

The first term $\psi^{(-)}$has a closed form solution that is not dependent on the constants $\Delta_{1}$ and $\Delta_{2}$, given

$$
\begin{aligned}
\psi^{(-)} & =\int_{0}^{\delta} \frac{b_{\mathrm{m}} \rho_{\mathrm{r}}}{1-b_{\mathrm{m}} \delta \rho_{\mathrm{r}}} \mathrm{d} \delta \\
& =-\ln \left(1-b_{\mathrm{m}} \delta \rho_{\mathrm{r}}\right) .
\end{aligned}
$$

The integral for $\psi^{(+)}$is more complicated; its form is given by

$$
\begin{aligned}
\psi^{(+)} & =\int_{0}^{\delta} \frac{\rho_{\mathrm{r}}}{\left(1+\Delta_{1} b_{\mathrm{m}} \delta \rho_{\mathrm{r}}\right)\left(1+\Delta_{2} b_{\mathrm{m}} \delta \rho_{\mathrm{r}}\right)} \mathrm{d} \delta \\
& =\frac{\ln \left(\frac{\Delta_{1} b_{\mathrm{m}} \rho_{\mathrm{r}} \delta+1}{\Delta_{2} b_{\mathrm{m}} \rho_{\mathrm{r}} \delta+1}\right)}{b_{\mathrm{m}}\left(\Delta_{1}-\Delta_{2}\right)},
\end{aligned}
$$

with the assumption that $\Delta_{1}-\Delta_{2} \neq 0$. This is true for SRK and PR, but not for the van der Waals equation of state, which has the constants $\Delta_{1}=\Delta_{2}=0$. For the van der Waals equation of state, setting $\Delta_{2}=0$, taking the limit as $\Delta_{1}$ approaches zero, and with the use of a single application of l'Hôpital's rule, it can be shown that

$$
\psi_{\mathrm{vdW}}^{(+)}=\rho_{\mathrm{r}} \delta
$$


Thus all three equations of state (SRK, PR, vdW) can be treated with a similar formulation.

In order to make use of the one-fluid model, derivatives with respect to $\tau$ and $\delta$ are required, for instance, in order to calculate the pressure as shown above. In this work, we provide a large number of analytic derivatives, including $\tau, \delta$, and composition partial derivatives. They were selected as the minimal set of derivatives needed to calculate critical points of binary mixtures with the use of entirely analytic derivatives.

\section{Derivatives at Constant Composition}

After having obtained the closed-form solution for the residual Helmholtz energy $\alpha^{\mathrm{r}}$ from Eq. (25), it is necessary to obtain several derivatives with respect to $\tau$ and $\delta$ at constant composition. The partial derivatives of $\alpha^{\mathrm{r}}$ at constant composition can be expressed in a compact form

$$
\left.\frac{\partial^{n+q} \alpha^{\mathrm{r}}}{\partial \delta^{n} \partial \tau^{q}}\right|_{\bar{x}}=\left.\frac{\partial^{n+q} \psi^{(-)}}{\partial \delta^{n} \partial \tau^{q}}\right|_{\bar{x}}-\left.\left.\frac{1}{R T_{\mathrm{r}}} \frac{\partial^{q}\left[\tau a_{\mathrm{m}}(\tau)\right]}{\partial \tau^{q}}\right|_{\bar{x}} \frac{\partial^{n} \psi^{(+)}}{\partial \delta^{n}}\right|_{\bar{x}}
$$

where $\bar{x}$ is the composition vector, and where the derivatives of the product of $\tau a_{\mathrm{m}}(\tau)$ can be expressed in the formulation

$$
\left.\frac{\partial^{n}\left[\tau a_{\mathrm{m}}(\tau)\right]}{\partial \tau^{n}}\right|_{\bar{x}}=\left.\tau \frac{\partial^{n}\left[a_{\mathrm{m}}(\tau)\right]}{\partial \tau^{n}}\right|_{\bar{x}}+\left.n \frac{\partial^{n-1}\left[a_{\mathrm{m}}(\tau)\right]}{\partial \tau^{n-1}}\right|_{\bar{x}},
$$

which is simply the $n$-th order partial derivative of a product. All 0 -th order partial derivatives are given by

$$
\left.\frac{\partial^{0} Y}{\partial \tau^{0}}\right|_{\bar{x}}=Y
$$

For example, the first partial derivative of $\tau a_{\mathrm{m}}(\tau)$ with respect to $\tau$ at constant composition would be equal to

$$
\left.\frac{\partial\left[\tau a_{\mathrm{m}}(\tau)\right]}{\partial \tau}\right|_{\bar{x}}=\left.\tau \frac{\partial\left[a_{\mathrm{m}}(\tau)\right]}{\partial \tau}\right|_{\bar{x}}+a_{\mathrm{m}}(\tau) .
$$

Partial derivatives of $\psi^{(-)}$and $\psi^{(+)}$will be discussed in the following sections.

Similarly to the 0 -th order derivatives with respect to $\tau$, all 0 -th order derivatives with respect to $\delta$ are given by

$$
\left.\frac{\partial^{0} Y}{\partial \delta^{0}}\right|_{\bar{x}}=Y
$$

\subsection{Derivatives of $\psi^{(-)}$}

The term $\psi^{(-)}$as given above in Eq. (27) is a function of $\delta$ and not a function of $\tau$ (though $b_{\mathrm{m}}$ is still a function of composition), therefore the first four derivatives of $\psi^{(-)}$with respect to $\delta$ at constant composition are

$$
\begin{aligned}
\psi^{(-)} & =-\ln \left(1-b_{\mathrm{m}} \delta \rho_{\mathrm{r}}\right) \\
\frac{\partial \psi^{(-)}}{\partial \delta} & =\frac{b_{\mathrm{m}} \rho_{\mathrm{r}}}{1-b_{\mathrm{m}} \delta \rho_{\mathrm{r}}} \\
\frac{\partial^{2} \psi^{(-)}}{\partial \delta^{2}} & =\frac{b_{\mathrm{m}}{ }^{2} \rho_{\mathrm{r}}{ }^{2}}{\left(1-b_{\mathrm{m}} \delta \rho_{\mathrm{r}}\right)^{2}}
\end{aligned}
$$




$$
\begin{aligned}
& \frac{\partial^{3} \psi^{(-)}}{\partial \delta^{3}}=\frac{2 b_{\mathrm{m}}{ }^{3} \rho_{\mathrm{r}}{ }^{3}}{\left(1-b_{\mathrm{m}} \delta \rho_{\mathrm{r}}\right)^{3}} \\
& \frac{\partial^{4} \psi^{(-)}}{\partial \delta^{4}}=\frac{6 b_{\mathrm{m}}{ }^{4} \rho_{\mathrm{r}}{ }^{4}}{\left(1-b_{\mathrm{m}} \delta \rho_{\mathrm{r}}\right)^{4}} .
\end{aligned}
$$

The reader might be interested to confirm that $\frac{\partial \psi^{(-)}}{\partial \delta}$ is the same as the integrand in Eq. (26).

Any partial derivative of $\psi^{(-)}$involving at least one derivative with respect to $\tau$ at constant composition is zero, which can be alternatively expressed as

$$
\left.\frac{\partial^{n+q} \psi^{(-)}}{\partial \delta^{n} \partial \tau^{q}}\right|_{\bar{x}}=0 \text { if } q>0
$$

\subsection{Derivatives of $\psi^{(+)}$}

As given before, the equation for $\psi^{(+)}$is

$$
\psi^{(+)}=\frac{\ln \left(\frac{\Delta_{1} b_{\mathrm{m}} \rho_{\mathrm{r}} \delta+1}{\Delta_{2} b_{\mathrm{m}} \rho_{\mathrm{r}} \delta+1}\right)}{b_{\mathrm{m}}\left(\Delta_{1}-\Delta_{2}\right)}
$$

In order to simplify the derivatives of $\psi^{(+)}$from Eq. (29) with respect to $\delta$, we introduce the term

$$
\Pi_{12}=\left(1+\Delta_{1} b_{\mathrm{m}} \rho_{\mathrm{r}} \delta\right)\left(1+\Delta_{2} b_{\mathrm{m}} \rho_{\mathrm{r}} \delta\right) .
$$

The term $\Pi_{12}$ is the denominator of the integrand of Eq. (28). The partial derivatives of $\Pi_{12}$ with respect to $\delta$ are then needed. The first three partial derivatives are

$$
\begin{gathered}
\frac{\partial \Pi_{12}}{\partial \delta}=b_{\mathrm{m}} \rho_{\mathrm{r}}\left(2 \Delta_{1} \Delta_{2} b_{\mathrm{m}} \delta \rho_{\mathrm{r}}+\Delta_{1}+\Delta_{2}\right) \\
\frac{\partial^{2} \Pi_{12}}{\partial \delta^{2}}=2 \Delta_{1} \Delta_{2} b_{\mathrm{m}}^{2} \rho_{\mathrm{r}}^{2} \\
\frac{\partial^{3} \Pi_{12}}{\partial \delta^{3}}=0 .
\end{gathered}
$$

All further $\delta$ derivatives of $\Pi_{12}$ are equal to zero.

The first four derivatives of $\psi^{(+)}$with respect to $\delta$ can be expressed in terms of $\Pi_{12}$ and derivatives of $\Pi_{12}$ as

$$
\begin{gathered}
\frac{\partial \psi^{(+)}}{\partial \delta}=\frac{\rho_{\mathrm{r}}}{\Pi_{12}} \\
\frac{\partial^{2} \psi^{(+)}}{\partial \delta^{2}}=-\rho_{\mathrm{r}} \frac{\frac{\partial \Pi_{12}}{\partial \delta}}{\Pi_{12}^{2}} \\
\frac{\partial^{3} \psi^{(+)}}{\partial \delta^{3}}=\rho_{\mathrm{r}} \frac{-\Pi_{12} \frac{\partial^{2} \Pi_{12}}{\partial \delta^{2}}+2\left(\frac{\partial \Pi_{12}}{\partial \delta}\right)^{2}}{\Pi_{12}^{3}}
\end{gathered}
$$




$$
\frac{\partial^{4} \psi^{(+)}}{\partial \delta^{4}}=\rho_{\mathrm{r}} \frac{6 \Pi_{12} \frac{\partial \Pi_{12}}{\partial \delta} \frac{\partial^{2} \Pi_{12}}{\partial \delta^{2}}-6\left(\frac{\partial \Pi_{12}}{\partial \delta}\right)^{3}}{\Pi_{12}^{4}} .
$$

Note that $\frac{\partial^{3} \Pi_{12}}{\partial \delta^{3}}=0$, which removes a term in the numerator of $\frac{\partial^{4} \psi^{(+)}}{\partial \delta^{4}}$.

\subsection{Derivatives of $a_{\mathrm{m}}$ and $b_{\mathrm{m}}$}

The $n$-th partial derivative of $a_{\mathrm{m}}$ (from Eq. (10)) with respect to $\tau$ at constant composition can be given by

$$
\left(\frac{\partial^{n} a_{\mathrm{m}}}{\partial \tau^{n}}\right)_{\bar{x}}=\sum_{i} \sum_{j} x_{i} x_{j} \frac{\mathrm{d}^{n} a_{i j}}{\mathrm{~d} \tau^{n}}
$$

The term $b_{\mathrm{m}}$ has neither $\tau$ nor $\delta$ dependence, and therefore all derivatives of $b_{\mathrm{m}}$ other than composition derivatives are equal to zero.

\subsection{Derivatives of $a_{i j}$}

The term $a_{i j}(\tau)$ is described above and given by the form in Eq. (12), where the only modification here is that we express the equation with $\tau$ as the independent variable instead of $T$. In order to avoid a rapidly increasing number of terms in higher derivatives of $a_{i j}(\tau)$ with respect to $\tau$, a $u_{i j}(\tau)$ function is substituted, where the generic $u_{i j}$ function is given by

$$
u_{i j}(\tau)=a_{i i}(\tau) a_{j j}(\tau)
$$

and therefore

$$
a_{i j}(\tau)=\left(1-k_{i j}\right) \cdot \sqrt{u_{i j}(\tau)} .
$$

The advantage of this substitution is that all derivatives of $a_{i j}$ with respect to $\tau$ can be expressed in terms of $u_{i j}$ and its derivatives, resulting in derivatives with more compact analytic forms. The first four derivatives of $a_{i j}$ with respect to $\tau$ can be given by

$$
\begin{gathered}
\frac{\mathrm{d} a_{i j}}{\mathrm{~d} \tau}=\frac{1-k_{i j}}{2 \sqrt{u_{i j}}} \frac{\mathrm{d} u_{i j}}{\mathrm{~d} \tau} \\
\frac{\mathrm{d}^{2} a_{i j}}{\mathrm{~d} \tau^{2}}=\frac{1-k_{i j}}{4 u_{i j}^{\frac{3}{2}}}\left(2 u_{i j} \frac{\mathrm{d}^{2} u_{i j}}{\mathrm{~d} \tau^{2}}-\left(\frac{\mathrm{d} u_{i j}}{\mathrm{~d} \tau}\right)^{2}\right) \\
\frac{\mathrm{d}^{3} a_{i j}}{\mathrm{~d} \tau^{3}}=\frac{1-k_{i j}}{8 u_{i j}^{\frac{5}{2}}}\left(4 u_{i j}^{2} \frac{\mathrm{d}^{3} u_{i j}}{\mathrm{~d} \tau^{3}}-6 u_{i j} \frac{\mathrm{d} u_{i j}}{\mathrm{~d} \tau} \frac{\mathrm{d}^{2} u_{i j}}{\mathrm{~d} \tau^{2}}+3\left(\frac{\mathrm{d} u_{i j}}{\mathrm{~d} \tau}\right)^{3}\right)
\end{gathered}
$$




$$
\frac{\mathrm{d}^{4} a_{i j}}{\mathrm{~d} \tau^{4}}=\frac{1-k_{i j}}{16 u_{i j}^{\frac{7}{2}}}\left(\begin{array}{c}
-4\left(4 \frac{\mathrm{d} u_{i j}}{\mathrm{~d} \tau} \frac{\mathrm{d}^{3} u_{i j}}{\mathrm{~d} \tau^{3}}+3\left(\frac{\mathrm{d}^{2} u_{i j}}{\mathrm{~d} \tau^{2}}\right)^{2}\right) u_{i j}^{2} \\
+8 u_{i j}^{3} \frac{\mathrm{d}^{4} u_{i j}}{\mathrm{~d} \tau^{4}}+36 u_{i j}\left(\frac{\mathrm{d} u_{i j}}{\mathrm{~d} \tau}\right)^{2} \frac{\mathrm{d}^{2} u_{i j}}{\mathrm{~d} \tau^{2}} \\
-15\left(\frac{\mathrm{d} u_{i j}}{\mathrm{~d} \tau}\right)^{4}
\end{array}\right)
$$

where we have assumed that $k_{i j}$ is a constant and not a function of temperature or composition. The first four derivatives of $u_{i j}$ with respect to $\tau$ can be given by

$$
\begin{gathered}
u_{i j}=a_{i i} a_{j j} \\
\frac{\mathrm{d} u_{i j}}{\mathrm{~d} \tau}=a_{i i} \frac{\mathrm{d} a_{j j}}{\mathrm{~d} \tau}+a_{j j} \frac{\mathrm{d} a_{i i}}{\mathrm{~d} \tau} \\
\frac{\mathrm{d}^{2} u_{i j}}{\mathrm{~d} \tau^{2}}=a_{i i} \frac{\mathrm{d}^{2} a_{j j}}{\mathrm{~d} \tau^{2}}+2 \frac{\mathrm{d} a_{i i}}{\mathrm{~d} \tau} \frac{\mathrm{d} a_{j j}}{\mathrm{~d} \tau}+a_{j j} \frac{\mathrm{d}^{2} a_{i i}}{\mathrm{~d} \tau^{2}} \\
\frac{\mathrm{d}^{3} u_{i j}}{\mathrm{~d} \tau^{3}}=a_{i i} \frac{\mathrm{d}^{3} a_{j j}}{\mathrm{~d} \tau^{3}}+3 \frac{\mathrm{d} a_{i i}}{\mathrm{~d} \tau} \frac{\mathrm{d}^{2} a_{i j}}{\mathrm{~d} \tau^{2}}+3 \frac{\mathrm{d}^{2} a_{i i}}{\mathrm{~d} \tau^{2}} \frac{\mathrm{d} a_{j j}}{\mathrm{~d} \tau}+a_{j j} \frac{\mathrm{d}^{3} a_{i i}}{\mathrm{~d} \tau^{3}} \\
\frac{\mathrm{d}^{4} u_{i j}}{\mathrm{~d} \tau^{4}}=a_{i i} \frac{\mathrm{d}^{4} a_{j j}}{\mathrm{~d} \tau^{4}}+4 \frac{\mathrm{d} a_{i i}}{\mathrm{~d} \tau} \frac{\mathrm{d}^{3} a_{j j}}{\mathrm{~d} \tau^{3}}+6 \frac{\mathrm{d}^{2} a_{i i}}{\mathrm{~d} \tau^{2}} \frac{\mathrm{d}^{2} a_{j j}}{\mathrm{~d} \tau^{2}}+4 \frac{\mathrm{d}^{3} a_{i i}}{\mathrm{~d} \tau^{3}} \frac{\mathrm{d} a_{i j}}{\mathrm{~d} \tau}+a_{j j} \frac{\mathrm{d}^{4} a_{i i}}{\mathrm{~d} \tau^{4}}
\end{gathered}
$$

\subsection{Derivatives of $a_{i i}(\tau)$}

The term $a_{i i}$ introduced in Eq. (14) can be expressed as $a_{i i}=a_{0, i i} B_{i}^{2}$, where $a_{0, i i}$ is a constant term dependent on the fluid and the equation of state (given in Table 1 ), and the term $B_{i}$ is given by

$$
B_{i}=1+\sum_{n=1}^{3} C_{n, i} D_{i}^{n},
$$

where the intermediate term $D_{i}$ (introduced to simplify derivatives of $a_{i i}$ after the substitution $T=T_{\mathrm{r}} / \tau$ ) is defined by

$$
D_{i}(\tau)=1-\sqrt{\frac{T_{\mathrm{r}}}{T_{\mathrm{c}, i}}} \cdot \frac{1}{\sqrt{\tau}}
$$

We then obtain the derivatives of $D_{i}$ from

$$
\begin{gathered}
\frac{\mathrm{d} D_{i}}{\mathrm{~d} \tau}=\frac{\sqrt{\frac{T_{\mathrm{r}}}{T_{\mathrm{c}, i}}}}{2 \tau^{\frac{3}{2}}} \\
\frac{\mathrm{d}^{2} D_{i}}{\mathrm{~d} \tau^{2}}=-\frac{3 \sqrt{\frac{T_{\mathrm{r}}}{T_{\mathrm{c}, i}}}}{4 \tau^{\frac{5}{2}}}
\end{gathered}
$$




$$
\begin{gathered}
\frac{\mathrm{d}^{3} D_{i}}{\mathrm{~d} \tau^{3}}=\frac{15 \sqrt{\frac{T_{\mathrm{r}}}{T_{\mathrm{c}, i}}}}{8 \tau^{\frac{7}{2}}} \\
\frac{\mathrm{d}^{4} D_{i}}{\mathrm{~d} \tau^{4}}=-\frac{105 \sqrt{\frac{T_{\mathrm{r}}}{T_{\mathrm{c}, i}}}}{16 \tau^{\frac{9}{2}}},
\end{gathered}
$$

with the derivatives of $B_{i}$ from

$$
\begin{aligned}
& \frac{\mathrm{d} B_{i}}{\mathrm{~d} \tau}=\sum_{n=1}^{3} n C_{n, i} D_{i}^{n-1} \frac{\mathrm{d} D_{i}}{\mathrm{~d} \tau} \\
& \frac{\mathrm{d}^{2} B_{i}}{\mathrm{~d} \tau^{2}}=\sum_{n=1}^{3} n C_{n, i}\left((n-1)\left(\frac{\mathrm{d} D_{i}}{\mathrm{~d} \tau}\right)^{2}+D_{i} \frac{\mathrm{d}^{2} D_{i}}{\mathrm{~d} \tau^{2}}\right) D_{i}^{n-2} \\
& \frac{\mathrm{d}^{3} B_{i}}{\mathrm{~d} \tau^{3}}=\sum_{n=1}^{3} n C_{n, i}\left(3(n-1) D_{i} \frac{\mathrm{d} D_{i}}{\mathrm{~d} \tau} \frac{\mathrm{d}^{2} D_{i}}{\mathrm{~d} \tau^{2}}+\left(n^{2}-3 n+2\right)\left(\frac{\mathrm{d} D_{i}}{\mathrm{~d} \tau}\right)^{3}+D_{i}^{2} \frac{\mathrm{d}^{3} D_{i}}{\mathrm{~d} \tau^{3}}\right) D_{i}^{n-3} \\
& \frac{\mathrm{d}^{4} B_{i}}{\mathrm{~d} \tau^{4}}=\sum_{n=1}^{3} n C_{n, i}\left(\begin{array}{l}
\left.6\left(n^{2}-3 n+2\right) D_{i}\left(\frac{\mathrm{d} D_{i}}{\mathrm{~d} \tau}\right)^{2} \frac{\mathrm{d}^{2} D_{i}}{\mathrm{~d} \tau^{2}}+\left(n^{3}-6 n^{2}+11 n-6\right)\left(\frac{\mathrm{d} D_{i}}{\mathrm{~d} \tau}\right)^{4}\right) \\
+\left(4(n-1) \frac{\mathrm{d} D_{i}}{\mathrm{~d} \tau} \frac{\mathrm{d}^{3} D_{i}}{\mathrm{~d} \tau^{3}}+3(n-1)\left(\frac{\mathrm{d}^{2} D_{i}}{\mathrm{~d} \tau^{2}}\right)^{2}\right) D_{i}^{2}+D_{i}^{3} \frac{\mathrm{d}^{4} D_{i}}{\mathrm{~d} \tau^{4}}
\end{array}\right) D_{i}^{n-4},
\end{aligned}
$$

and finally the derivatives of $a_{i i}$ from

$$
\begin{gathered}
\frac{\mathrm{d} a_{i i}}{\mathrm{~d} \tau}=2 a_{0, i i} B_{i} \frac{\mathrm{d} B_{i}}{\mathrm{~d} \tau} \\
\frac{\mathrm{d}^{2} a_{i i}}{\mathrm{~d} \tau^{2}}=2 a_{0, i i}\left(B_{i} \frac{\mathrm{d}^{2} B_{i}}{\mathrm{~d} \tau^{2}}+\left(\frac{\mathrm{d} B_{i}}{\mathrm{~d} \tau}\right)^{2}\right) \\
\frac{\mathrm{d}^{3} a_{i i}}{\mathrm{~d} \tau^{3}}=2 a_{0, i i}\left(B_{i} \frac{\mathrm{d}^{3} B_{i}}{\mathrm{~d} \tau^{3}}+3 \frac{\mathrm{d} B_{i}}{\mathrm{~d} \tau} \frac{\mathrm{d}^{2} B_{i}}{\mathrm{~d} \tau^{2}}\right) \\
\frac{\mathrm{d}^{4} a_{i i}}{\mathrm{~d} \tau^{4}}=2 a_{0, i i}\left(B_{i} \frac{\mathrm{d}^{4} B_{i}}{\mathrm{~d} \tau^{4}}+4 \frac{\mathrm{d} B_{i}}{\mathrm{~d} \tau} \frac{\mathrm{d}^{3} B_{i}}{\mathrm{~d} \tau^{3}}+3\left(\frac{\mathrm{d}^{2} B_{i}}{\mathrm{~d} \tau^{2}}\right)^{2}\right) .
\end{gathered}
$$

In the case that $C_{1, i}=m_{i i}, C_{2, i}=0$, and $C_{3, i}=0$, the derivatives of $a_{i i}$ are significantly simplified, and these simplified derivatives are presented in the supplementary information for completeness.

\section{Composition Derivatives}

The non-dimensionalized residual Helmholtz energy $\alpha^{\mathrm{r}}$ is given by Eq. (25). In order to make use of the one-fluid model for carrying out mixture calculations like vapor-liquid equilibria, or to calculate critical points with the use of analytic derivatives, a large number of composition derivatives is required. 
The first three composition derivatives of this term are given by

$$
\begin{aligned}
& \frac{\partial}{\partial x_{i}}\left[\left.\frac{\partial^{n+q} \alpha^{\mathrm{r}}}{\partial \delta^{n} \partial \tau^{q}}\right|_{\bar{x}}\right]_{x_{j}}=\frac{\partial}{\partial x_{i}}\left[\left.\frac{\partial^{n+q} \psi^{(-)}}{\partial \delta^{n} \partial \tau^{q}}\right|_{\bar{x}}\right]_{x_{j}}-\frac{1}{R T_{\mathrm{r}}}\left[\begin{array}{c}
\left.\frac{\partial^{q+1}\left[\tau a_{\mathrm{m}}(\tau)\right]}{\partial \tau^{q} \partial x_{i}} \frac{\partial^{n} \psi^{(+)}}{\partial \delta^{n}}\right|_{\bar{x}} \\
+\left.\frac{\partial^{q}\left[\tau a_{\mathrm{m}}(\tau)\right]}{\partial \tau^{q}}\right|_{\bar{x}} \frac{\partial^{n+1} \psi^{(+)}}{\partial \delta^{n} \partial x_{i}}
\end{array}\right]
\end{aligned}
$$

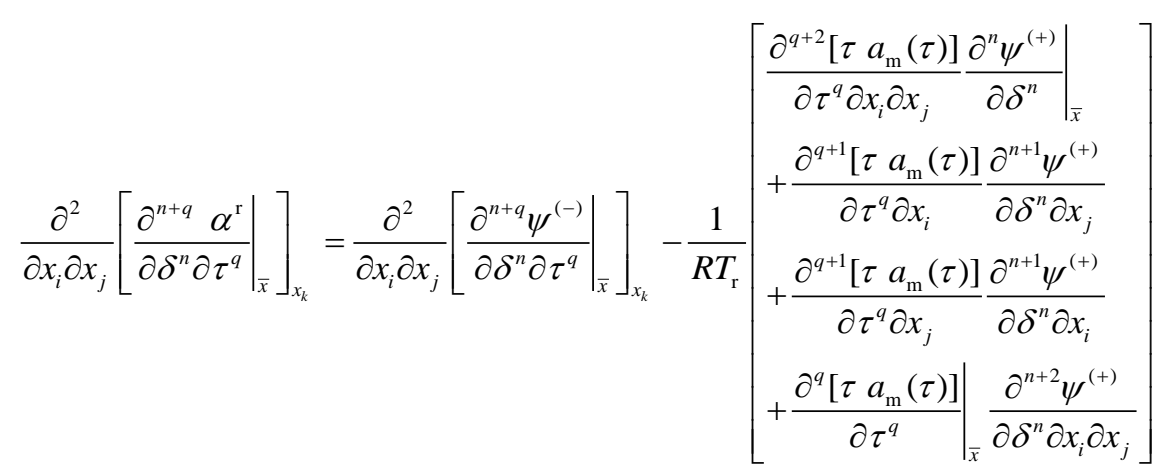

$$
\begin{aligned}
& \left.\frac{\partial^{q+3}\left[\tau a_{\mathrm{m}}(\tau)\right]}{\partial \tau^{q} \partial x_{i} \partial x_{j} \partial x_{k}} \frac{\partial^{n} \psi^{(+)}}{\partial \delta^{n}}\right|_{\bar{x}} \\
& +\frac{\partial^{q+2}\left[\tau a_{\mathrm{m}}(\tau)\right]}{\partial \tau^{q} \partial x_{i} \partial x_{j}} \frac{\partial^{n+1} \psi^{(+)}}{\partial \delta^{n} \partial x_{k}} \\
& +\frac{\partial^{q+2}\left[\tau a_{m}(\tau)\right]}{\partial \tau^{q} \partial x_{i} \partial x_{k}} \frac{\partial^{n+1} \psi^{(+)}}{\partial \delta^{n} \partial x_{j}} \\
& +\frac{\partial^{q+1}\left[\tau a_{\mathrm{m}}(\tau)\right]}{\partial \tau^{q} \partial x_{i}} \frac{\partial^{n+2} \psi^{(+)}}{\partial \delta^{n} \partial x_{j} \partial x_{k}} \\
& +\frac{\partial^{q+2}\left[\tau a_{m}(\tau)\right]}{\partial \tau^{q} \partial x_{j} \partial x_{k}} \frac{\partial^{n+1} \psi^{(+)}}{\partial \delta^{n} \partial x_{i}} \\
& +\frac{\partial^{q+1}\left[\tau a_{\mathrm{m}}(\tau)\right]}{\partial \tau^{q} \partial x_{j}} \frac{\partial^{n+2} \psi^{(+)}}{\partial \delta^{n} \partial x_{i} \partial x_{k}} \\
& +\frac{\partial^{q+1}\left[\tau a_{m}(\tau)\right]}{\partial \tau^{q} \partial x_{k}} \frac{\partial^{n+2} \psi^{(+)}}{\partial \delta^{n} \partial x_{i} \partial x_{j}} \\
& {\left[+\left.\frac{\partial^{q}\left[\tau a_{\mathrm{m}}(\tau)\right]}{\partial \tau^{q}}\right|_{\bar{x}} \frac{\partial^{n+3} \psi^{(+)}}{\partial \delta^{n} \partial x_{i} \partial x_{j} \partial x_{k}}\right]}
\end{aligned}
$$

The individual derivative terms involved in each derivative are covered in the following sections.

\subsection{Composition Derivatives of $\psi^{(-)}$}

The derivatives of $\psi^{(-)}$from Eq. (27) with respect to $\delta$ and one composition derivative with respect to $x_{i}$ with all other mole fractions held constant are given by 


$$
\begin{gathered}
\frac{\partial \psi^{(-)}}{\partial x_{i}}=\frac{\delta \rho_{\mathrm{r}} \frac{\partial b_{\mathrm{m}}}{\partial x_{i}}}{1-\delta \rho_{\mathrm{r}} b_{\mathrm{m}}} \\
\frac{\partial^{2} \psi^{(-)}}{\partial x_{i} \partial \delta}=\frac{\rho_{\mathrm{r}} \frac{\partial b_{\mathrm{m}}}{\partial x_{i}}}{\left(1-\delta \rho_{\mathrm{r}} b_{\mathrm{m}}\right)^{2}} \\
\frac{\partial^{3} \psi^{(-)}}{\partial x_{i} \partial \delta^{2}}=\frac{2 \rho_{\mathrm{r}}^{2} b_{\mathrm{m}} \frac{\partial b_{\mathrm{m}}}{\partial x_{i}}}{\left(1-\delta \rho_{\mathrm{r}} b_{\mathrm{m}}\right)^{3}} \\
\frac{\partial^{4} \psi^{(-)}}{\partial x_{i} \partial \delta^{3}}=\frac{6 \rho_{\mathrm{r}}^{3} b_{\mathrm{m}}^{2} \frac{\partial b_{\mathrm{m}}}{\partial x_{i}}}{\left(1-\delta \rho_{\mathrm{r}} b_{\mathrm{m}}\right)^{4}} \\
\frac{\partial^{5} \psi^{(-)}}{\partial x_{i} \partial \delta^{4}}=\frac{24 \rho_{\mathrm{r}}^{4} b_{\mathrm{m}}^{3} \frac{\partial b_{\mathrm{m}}}{\partial x_{i}}}{\left(1-\delta \rho_{\mathrm{r}} b_{\mathrm{m}}\right)^{5}} .
\end{gathered}
$$

The derivatives of $\psi^{(-)}$with respect to $\delta$ and two composition derivatives are given by

$$
\begin{gathered}
\frac{\partial^{2} \psi^{(-)}}{\partial x_{i} \partial x_{j}}=\frac{\delta \rho_{\mathrm{r}} \frac{\partial^{2} b_{\mathrm{m}}}{\partial x_{i} \partial x_{j}}}{1-\delta \rho_{\mathrm{r}} b_{\mathrm{m}}}+\frac{\delta^{2} \rho_{\mathrm{r}}^{2} \frac{\partial b_{\mathrm{m}}}{\partial x_{i}} \frac{\partial b_{\mathrm{m}}}{\partial x_{j}}}{\left(1-\delta \rho_{\mathrm{r}} b_{\mathrm{m}}\right)^{2}} \\
\frac{\partial^{3} \psi^{(-)}}{\partial x_{i} \partial x_{j} \partial \delta}=\frac{\rho_{\mathrm{r}} \frac{\partial^{2} b_{\mathrm{m}}}{\partial x_{i} \partial x_{j}}}{\left(1-\delta \rho_{\mathrm{r}} b_{\mathrm{m}}\right)^{2}}+\frac{2 \delta \rho_{\mathrm{r}}^{2} \frac{\partial b_{\mathrm{m}}}{\partial x_{i}} \frac{\partial b_{\mathrm{m}}}{\partial x_{j}}}{\left(1-\delta \rho_{\mathrm{r}} b_{\mathrm{m}}\right)^{3}} \\
\frac{\partial^{4} \psi^{(-)}}{\partial x_{i} \partial x_{j} \partial \delta^{2}}=\frac{2 \rho_{\mathrm{r}}^{2} b_{\mathrm{m}} \frac{\partial^{2} b_{\mathrm{m}}}{\partial x_{i} \partial x_{j}}}{\left(1-\delta \rho_{\mathrm{r}} b_{\mathrm{m}}\right)^{3}}+\frac{2 \rho_{\mathrm{r}}^{2} \frac{\partial b_{\mathrm{m}}}{\partial x_{i}} \frac{\partial b_{\mathrm{m}}}{\partial x_{j}}}{\left(1-\delta \rho_{\mathrm{r}} b_{\mathrm{m}}\right)^{4}}\left(2 \delta \rho_{\mathrm{r}} b_{\mathrm{m}}+1\right) \\
\frac{\partial^{5} \psi^{(-)} \partial x_{j} \partial \delta^{3}}{2}=\frac{\left(1-\delta \rho_{\mathrm{r}}^{2} \frac{\partial^{2} b_{\mathrm{m}}}{\partial x_{i} \partial x_{j}}\right.}{\left(1-\rho_{\mathrm{r}}\right.}+\frac{12 \rho_{\mathrm{r}}^{3} \frac{\partial b_{\mathrm{m}}}{\partial x_{i}} \frac{\partial b_{\mathrm{m}}}{\partial x_{j}}}{\left(1-\delta \rho_{\mathrm{r}} b_{\mathrm{m}}\right)^{5}}\left(\delta \rho_{\mathrm{r}} b_{\mathrm{m}}+1\right) \\
\frac{\partial^{6} \psi^{(-)}}{\partial x_{i} \partial x_{j} \partial \delta^{4}}=\frac{24 \rho_{\mathrm{r}}^{4} b_{\mathrm{m}}^{3} \frac{\partial^{2} b_{\mathrm{m}}}{\partial x_{i} \partial x_{j}}}{\left(1-\delta \rho_{\mathrm{r}} b_{\mathrm{m}}\right)^{5}}+\frac{24 \rho_{\mathrm{r}}^{4} b_{\mathrm{m}}^{2} \frac{\partial b_{\mathrm{m}}}{\partial x_{i}} \frac{\partial b_{\mathrm{m}}}{\partial x_{j}}}{\left(1-\delta \rho_{\mathrm{r}} b_{\mathrm{m}}\right)^{6}}\left(2 \delta \rho_{\mathrm{r}} b_{\mathrm{m}}+3\right) .
\end{gathered}
$$

The derivatives of $\psi^{(-)}$with respect to $\delta$ and three composition derivatives are given by 


$$
\begin{aligned}
& \frac{\partial^{3} \psi^{(-)}}{\partial x_{i} \partial x_{j} \partial x_{k}}=\left[\begin{array}{l}
\frac{\delta \rho_{\mathrm{r}} \frac{\partial^{3} b_{\mathrm{m}}}{\partial x_{i} \partial x_{j} \partial x_{k}}}{1-\delta \rho_{\mathrm{r}} b_{\mathrm{m}}} \\
+\frac{2 \delta^{3} \rho_{\mathrm{r}}^{3} \frac{\partial b_{\mathrm{m}}}{\partial x_{i}} \frac{\partial b_{\mathrm{m}}}{\partial x_{j}} \frac{\partial b_{\mathrm{m}}}{\partial x_{k}}}{\left(1-\delta \rho_{\mathrm{r}} b_{\mathrm{m}}\right)^{3}} \\
+\left(\begin{array}{c}
\frac{\partial b_{\mathrm{m}}}{\partial x_{i}} \frac{\partial^{2} b_{\mathrm{m}}}{\partial x_{j} \partial x_{k}} \\
+\frac{\partial b_{\mathrm{m}}}{\delta^{2} \rho_{\mathrm{r}}^{2}} \frac{\partial^{2} b_{\mathrm{m}}}{\partial x_{j}} \\
\frac{\partial x_{i} \partial x_{k}}{\left(1-\delta \rho_{\mathrm{r}} b_{\mathrm{m}}\right)^{2}} \\
+\frac{\partial b_{\mathrm{m}}}{\partial x_{k}} \frac{\partial^{2} b_{\mathrm{m}}}{\partial x_{i} \partial x_{j}}
\end{array}\right)
\end{array}\right] \\
& \frac{\partial^{4} \psi^{(-)}}{\partial x_{i} \partial x_{j} \partial x_{k} \partial \delta}=\left[\begin{array}{l}
\frac{\rho_{\mathrm{r}} \frac{\partial^{3} b_{\mathrm{m}}}{\partial x_{i} \partial x_{j} \partial x_{k}}}{\left(1-\delta \rho_{\mathrm{r}} b_{\mathrm{m}}\right)^{2}} \\
6 \delta^{2} \rho_{\mathrm{r}}^{3} \frac{\partial b_{\mathrm{m}}}{\partial x_{i}} \frac{\partial b_{\mathrm{m}} \partial x_{j} \partial x_{\mathrm{m}}}{\left(1-\delta \rho_{\mathrm{r}} b_{\mathrm{m}}\right)^{4}} \\
+\left(\begin{array}{c}
\frac{\partial b_{\mathrm{m}}}{\partial x_{i}} \frac{\partial^{2} b_{\mathrm{m}}}{\partial x_{j} \partial x_{k}} \\
+\frac{\partial b_{\mathrm{m}}}{\partial x_{j}} \frac{\partial^{2} b_{\mathrm{m}}}{\partial x_{i} \partial x_{k}} \\
+\frac{\partial b_{\mathrm{m}}}{\partial x_{k}} \frac{\partial^{2} b_{\mathrm{m}}}{\partial x_{i} \partial x_{j}}
\end{array}\right)
\end{array}\right]
\end{aligned}
$$

\subsection{Composition Derivatives of $\Pi_{12}$}

As given in Eq. (43), the intermediate term $\Pi_{12}$ is given by

$$
\Pi_{12}=\left(1+\Delta_{1} b_{\mathrm{m}} \rho_{\mathrm{r}} \delta\right)\left(1+\Delta_{2} b_{\mathrm{m}} \rho_{\mathrm{r}} \delta\right) .
$$

This intermediate term was introduced in order to yield more compact derivative forms for the derivatives of $\psi^{(+)}$. The first composition derivatives with up to four $\delta$ derivatives and all other mole fractions held constant are given by

$$
\begin{gathered}
\frac{\partial \Pi_{12}}{\partial x_{i}}=\delta \rho_{\mathrm{r}} \frac{\partial b_{\mathrm{m}}}{\partial x_{i}}\left[2 \Delta_{1} \Delta_{2} \delta \rho_{\mathrm{r}} b_{\mathrm{m}}+\Delta_{1}+\Delta_{2}\right] \\
\frac{\partial^{2} \Pi_{12}}{\partial x_{i} \partial \delta}=\rho_{\mathrm{r}} \frac{\partial b_{\mathrm{m}}}{\partial x_{i}}\left[4 \Delta_{1} \Delta_{2} \delta \rho_{\mathrm{r}} b_{\mathrm{m}}+\Delta_{1}+\Delta_{2}\right] \\
\frac{\partial^{3} \Pi_{12}}{\partial x_{i} \partial \delta^{2}}=4 \Delta_{1} \Delta_{2} \rho_{\mathrm{r}}^{2} b_{\mathrm{m}} \frac{\partial b_{\mathrm{m}}}{\partial x_{i}}
\end{gathered}
$$




$$
\frac{\partial^{4} \Pi_{12}}{\partial x_{i} \partial \delta^{3}}=\frac{\partial^{5} \Pi_{12}}{\partial x_{i} \partial \delta^{4}}=0
$$

The second cross composition derivatives with up to four $\delta$ derivatives and all other mole fractions held constant are given by

$$
\begin{gathered}
\frac{\partial^{2} \Pi_{12}}{\partial x_{i} \partial x_{j}}=\delta \rho_{\mathrm{r}}\left(2 \Delta_{1} \Delta_{2} \delta \rho_{\mathrm{r}} \frac{\partial b_{\mathrm{m}}}{\partial x_{i}} \frac{\partial b_{\mathrm{m}}}{\partial x_{j}}+\left(2 \Delta_{1} \Delta_{2} \delta \rho_{\mathrm{r}} b_{\mathrm{m}}+\Delta_{1}+\Delta_{2}\right) \frac{\partial^{2} b_{\mathrm{m}}}{\partial x_{i} \partial x_{j}}\right) \\
\frac{\partial^{3} \Pi_{12}}{\partial x_{i} \partial x_{j} \partial \delta}=\rho_{\mathrm{r}}\left(4 \Delta_{1} \Delta_{2} \delta \rho_{\mathrm{r}} \frac{\partial b_{\mathrm{m}}}{\partial x_{i}} \frac{\partial b_{\mathrm{m}}}{\partial x_{j}}+\left(4 \Delta_{1} \Delta_{2} \delta \rho_{\mathrm{r}} b_{\mathrm{m}}+\Delta_{1}+\Delta_{2}\right) \frac{\partial^{2} b_{\mathrm{m}}}{\partial x_{i} \partial x_{j}}\right) \\
\frac{\partial^{4} \Pi_{12}}{\partial x_{i} \partial x_{j} \partial \delta^{2}}=4 \Delta_{1} \Delta_{2} \rho_{\mathrm{r}}^{2}\left(b_{\mathrm{m}} \frac{\partial^{2} b_{\mathrm{m}}}{\partial x_{i} \partial x_{j}}+\frac{\partial b_{\mathrm{m}}}{\partial x_{i}} \frac{\partial b_{\mathrm{m}}}{\partial x_{j}}\right) \\
\frac{\partial^{5} \Pi_{12}}{\partial x_{i} \partial x_{j} \partial \delta^{3}}=\frac{\partial^{6} \Pi_{12}}{\partial x_{i} \partial x_{j} \partial \delta^{4}}=0 .
\end{gathered}
$$

The third composition derivative is given by

$$
\left.\frac{\partial^{3} \Pi_{12}}{\partial x_{i} \partial x_{j} \partial x_{k}}=\delta \rho_{\mathrm{r}}\left(2 \Delta_{1} \Delta_{2} \delta \rho_{\mathrm{r}} b_{\mathrm{m}}+\Delta_{1}+\Delta_{2}\right) \frac{\partial^{3} b_{\mathrm{m}}}{\partial x_{i} \partial x_{j} \partial x_{k}}+2 \Delta_{1} \Delta_{2} \delta \rho_{\mathrm{r}}\left(\begin{array}{c}
\frac{\partial b_{\mathrm{m}}}{\partial x_{i}} \frac{\partial^{2} b_{\mathrm{m}}}{\partial x_{j} \partial x_{k}} \\
+\frac{\partial b_{\mathrm{m}}}{\partial x_{j}} \frac{\partial^{2} b_{\mathrm{m}}}{\partial x_{i} \partial x_{k}} \\
+\frac{\partial b_{\mathrm{m}}}{\partial x_{k}} \frac{\partial^{2} b_{\mathrm{m}}}{\partial x_{i} \partial x_{j}}
\end{array}\right)\right)
$$

The fourth mixed derivative (three with respect to composition, and one with respect to $\delta$ ) is given by

$$
\frac{\partial^{4} \Pi_{12}}{\partial x_{i} \partial x_{j} \partial x_{k} \partial \delta}=\rho_{\mathrm{r}}\left(\left(4 \Delta_{1} \Delta_{2} \delta \rho_{\mathrm{r}} b_{\mathrm{m}}+\Delta_{1}+\Delta_{2}\right) \frac{\partial^{3} b_{\mathrm{m}}}{\partial x_{i} \partial x_{j} \partial x_{k}}+4 \Delta_{1} \Delta_{2} \delta \rho_{\mathrm{r}}\left(\begin{array}{c}
\frac{\partial b_{\mathrm{m}}}{\partial x_{i}} \frac{\partial^{2} b_{\mathrm{m}}}{\partial x_{j} \partial x_{k}} \\
+\frac{\partial b_{\mathrm{m}}}{\partial x_{j}} \frac{\partial^{2} b_{\mathrm{m}}}{\partial x_{i} \partial x_{k}} \\
+\frac{\partial b_{\mathrm{m}}}{\partial x_{k}} \frac{\partial^{2} b_{\mathrm{m}}}{\partial x_{i} \partial x_{j}}
\end{array}\right)\right) .
$$

\subsection{Composition Derivatives of $\psi^{(+)}$}

In order to simplify the composition derivatives of $\psi^{(+)}$, we introduce a variable $A$ (which is a function of composition and $\delta$ ) given by

$$
A=\ln \left(\frac{\delta \rho_{\mathrm{r}} b_{\mathrm{m}} \Delta_{1}+1}{\delta \rho_{\mathrm{r}} b_{\mathrm{m}} \Delta_{2}+1}\right)
$$

The first three composition derivatives of $A$ are 


$$
\begin{gathered}
\frac{\partial A}{\partial x_{i}}=\frac{\delta \rho_{\mathrm{r}} \frac{\partial b_{\mathrm{m}}}{\partial x_{i}}\left(\Delta_{1}-\Delta_{2}\right)}{\Pi_{12}} \\
\frac{\partial^{2} A}{\partial x_{i} \partial x_{j}}=\frac{\delta \rho_{\mathrm{r}}\left(\Delta_{1}-\Delta_{2}\right)}{\Pi_{12}^{2}}\left(\Pi_{12} \frac{\partial^{2} b_{\mathrm{m}}}{\partial x_{i} \partial x_{j}}-\frac{\partial \Pi_{12}}{\partial x_{j}} \frac{\partial b_{\mathrm{m}}}{\partial x_{i}}\right) \\
\left.\frac{\partial^{3} A}{\partial x_{i} \partial x_{j} \partial x_{k}}=\frac{\delta \rho_{\mathrm{r}}\left(\Delta_{1}-\Delta_{2}\right)}{\Pi_{12}^{3}}\left[\begin{array}{c}
\frac{\partial \Pi_{12}}{\partial x_{j}} \frac{\partial^{2} b_{\mathrm{m}}}{\partial x_{i} \partial x_{k}} \\
+\frac{\partial \Pi_{12}}{\partial x_{k}} \frac{\partial^{2} b_{\mathrm{m}}}{\partial x_{i} \partial x_{j}} \\
+\frac{\partial b_{\mathrm{m}}}{\partial x_{i}} \frac{\partial^{2} \Pi_{12}}{\partial x_{j} \partial x_{k}}
\end{array}\right)+\Pi_{12}^{2} \frac{\partial^{3} b_{\mathrm{m}}}{\partial x_{i} \partial x_{j} \partial x_{k}}+2 \frac{\partial \Pi_{12}}{\partial x_{j}} \frac{\partial \Pi_{12}}{\partial x_{k}} \frac{\partial b_{\mathrm{m}}}{\partial x_{i}}\right],
\end{gathered}
$$

where the parameter $\Pi_{12}$ is obtained from Eq. (43).

Furthermore, we introduce a term $c=1 / b_{\mathrm{m}}$, which has the composition derivatives given by

$$
\begin{gathered}
\frac{\partial c}{\partial x_{i}}=-\frac{\frac{\partial b_{\mathrm{m}}}{\partial x_{i}}}{b_{\mathrm{m}}^{2}} \\
\frac{\partial^{2} c}{\partial x_{i} \partial x_{j}}=\frac{1}{b_{\mathrm{m}}^{3}}\left(2 \frac{\partial b_{\mathrm{m}}}{\partial x_{i}} \frac{\partial b_{\mathrm{m}}}{\partial x_{j}}-b_{\mathrm{m}} \frac{\partial^{2} b_{\mathrm{m}}}{\partial x_{i} \partial x_{j}}\right)
\end{gathered}
$$

and

$$
\frac{\partial^{3} c}{\partial x_{i} \partial x_{j} \partial x_{k}}=\frac{1}{b_{\mathrm{m}}^{4}}\left(2 b_{\mathrm{m}}\left(\begin{array}{l}
\frac{\partial b_{\mathrm{m}}}{\partial x_{i}} \frac{\partial^{2} b_{\mathrm{m}}}{\partial x_{j} \partial x_{k}} \\
+\frac{\partial b_{\mathrm{m}}}{\partial x_{j}} \frac{\partial^{2} b_{\mathrm{m}}}{\partial x_{i} \partial x_{k}} \\
+\frac{\partial b_{\mathrm{m}}}{\partial x_{k}} \frac{\partial^{2} b_{\mathrm{m}}}{\partial x_{i} \partial x_{j}}
\end{array}\right)-b_{\mathrm{m}}^{2} \frac{\partial^{3} b_{\mathrm{m}}}{\partial x_{i} \partial x_{j} \partial x_{k}}-6 \frac{\partial b_{\mathrm{m}}}{\partial x_{i}} \frac{\partial b_{\mathrm{m}}}{\partial x_{j}} \frac{\partial b_{\mathrm{m}}}{\partial x_{k}}\right)
$$

With the use of the $A$ and $c$ parameters and their derivatives, the first two composition derivatives of $\psi^{(+)}$ (taken at constant $\delta$ ) can then be obtained from

$$
\psi^{(+)}=\frac{A c}{\Delta_{1}-\Delta_{2}}
$$

(which is equivalent to Eq. (29)), and further composition derivatives are obtained from

$$
\begin{gathered}
\frac{\partial \psi^{(+)}}{\partial x_{i}}=\frac{\left(A \frac{\partial c}{\partial x_{i}}+c \frac{\partial A}{\partial x_{i}}\right)}{\Delta_{1}-\Delta_{2}} \\
\frac{\partial^{2} \psi^{(+)}}{\partial x_{i} \partial x_{j}}=\frac{\left(A \frac{\partial^{2} c}{\partial x_{i} \partial x_{j}}+c \frac{\partial^{2} A}{\partial x_{i} \partial x_{j}}+\frac{\partial A}{\partial x_{i}} \frac{\partial c}{\partial x_{j}}+\frac{\partial A}{\partial x_{j}} \frac{\partial c}{\partial x_{i}}\right)}{\Delta_{1}-\Delta_{2}}
\end{gathered}
$$




$$
\frac{\partial^{3} \psi^{(+)}}{\partial x_{i} \partial x_{j} \partial x_{k}}=\frac{\left(\begin{array}{c}
A \frac{\partial^{3} c}{\partial x_{i} \partial x_{j} \partial x_{k}}+c \frac{\partial^{3} A}{\partial x_{i} \partial x_{j} \partial x_{k}} \\
+\frac{\partial A}{\partial x_{i}} \frac{\partial^{2} c}{\partial x_{j} \partial x_{k}}+\frac{\partial A}{\partial x_{j}} \frac{\partial^{2} c}{\partial x_{i} \partial x_{k}} \\
+\frac{\partial A}{\partial x_{k}} \frac{\partial^{2} c}{\partial x_{i} \partial x_{j}}+\frac{\partial c}{\partial x_{i}} \frac{\partial^{2} A}{\partial x_{j} \partial x_{k}} \\
+\frac{\partial c}{\partial x_{j}} \frac{\partial^{2} A}{\partial x_{i} \partial x_{k}}+\frac{\partial c}{\partial x_{k}} \frac{\partial^{2} A}{\partial x_{i} \partial x_{j}}
\end{array}\right)}{\Delta_{1}-\Delta_{2}}
$$

For mixed derivatives with one composition derivative, the first four $\delta$ derivatives of $\partial \psi^{(+)} / \partial x_{i}$ are given by the following equations

$$
\begin{gathered}
\frac{\partial^{2} \psi^{(+)}}{\partial x_{i} \partial \delta}=\frac{-\rho_{\mathrm{r}}}{\Pi_{12}^{2}} \frac{\partial \Pi_{12}}{\partial x_{i}} \\
\frac{\partial^{3} \psi^{(+)}}{\partial x_{i} \partial \delta^{2}}=\frac{-\rho_{\mathrm{r}}}{\Pi_{12}^{2}}\left[\frac{\partial^{2} \Pi_{12}}{\partial x_{i} \partial \delta}+\frac{2 \Pi_{12}}{\rho_{\mathrm{r}}} \frac{\partial \Pi_{12}}{\partial \delta} \frac{\partial^{2} \psi^{(+)}}{\partial x_{i} \partial \delta}\right] \\
\frac{\partial^{4} \psi^{(+)}}{\partial x_{i} \partial \delta^{3}}=\frac{-\rho_{\mathrm{r}}}{\Pi_{12}^{2}}\left[\frac{\partial^{3} \Pi_{12}}{\partial x_{i} \partial \delta^{2}}+\frac{2}{\rho_{\mathrm{r}}}\left[\left(\frac{\partial \Pi_{12}}{\partial \delta}\right)^{2}+\Pi_{12} \frac{\partial^{2} \Pi_{12}}{\partial \delta^{2}}\right] \frac{\partial^{2} \psi^{(+)}}{\partial x_{i} \partial \delta}+\frac{4}{\rho_{\mathrm{r}}} \Pi_{12} \frac{\partial \Pi_{12}}{\partial \delta} \frac{\partial^{3} \psi^{(+)}}{\partial x_{i} \partial \delta^{2}}\right] \\
\frac{\partial^{5} \psi^{(+)}}{\partial x_{i} \partial \delta^{4}}=\frac{-\rho_{\mathrm{r}}}{\Pi_{12}^{2}}\left[\frac{6}{\rho_{\mathrm{r}}} \frac{\partial \Pi_{12}}{\partial \delta} \frac{\partial^{2} \Pi_{12}}{\partial \delta^{2}} \frac{\partial^{2} \psi^{(+)}}{\partial x_{i} \partial \delta}+\frac{6}{\rho_{\mathrm{r}}}\left[\left(\frac{\partial \Pi_{12}}{\partial \delta}\right)^{2}+\Pi_{12} \frac{\partial^{2} \Pi_{12}}{\partial \delta^{2}}\right] \frac{\partial^{3} \psi^{(+)}}{\partial x_{i} \partial \delta^{2}}+\frac{6}{\rho_{\mathrm{r}}} \Pi_{12} \frac{\partial \Pi_{12}}{\partial \delta} \frac{\partial^{4} \psi^{(+)}}{\partial x_{i} \partial \delta^{3}}\right] . \\
\text { Note that } \frac{\partial^{4} \Pi_{12}}{\partial x_{i} \partial \delta^{3}}=0 \text { and } \frac{\partial^{3} \Pi_{12}}{\partial \delta^{3}}=0 \text {, which removes contributions from } \frac{\partial^{5} \psi^{(+)}}{\partial x_{i} \partial \delta^{4}} .
\end{gathered}
$$

The $\delta$ derivatives of the second and higher mixed composition derivatives are given by the equations

$$
\begin{aligned}
& \frac{\partial^{3} \psi^{(+)}}{\partial x_{i} \partial x_{j} \partial \delta}=-\frac{1}{\Pi_{12}^{2}}\left(\rho_{\mathrm{r}} \frac{\partial^{2} \Pi_{12}}{\partial x_{i} \partial x_{j}}+2 \Pi_{12} \frac{\partial \Pi_{12}}{\partial x_{j}} \frac{\partial^{2} \psi^{(+)}}{\partial \delta \partial x_{i}}\right)
\end{aligned}
$$

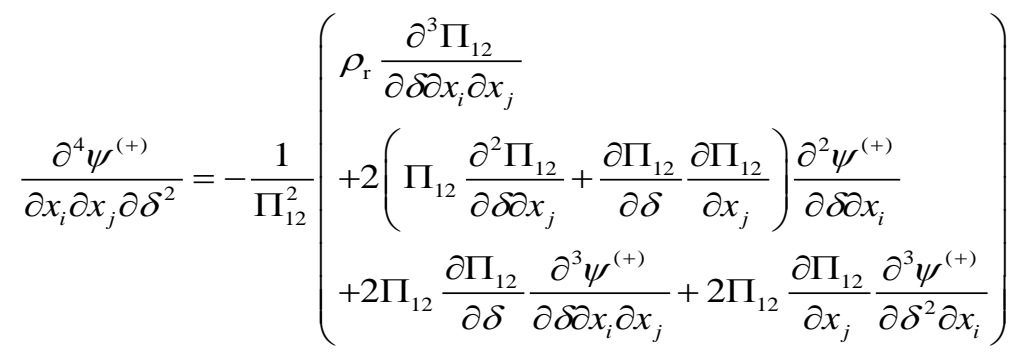




$$
\begin{aligned}
& \rho_{\mathrm{r}} \frac{\partial^{4} \Pi_{12}}{\partial \delta^{2} \partial x_{i} \partial x_{j}} \\
& +2\left(\Pi_{12} \frac{\partial^{2} \Pi_{12}}{\partial \delta^{2}}+\left(\frac{\partial \Pi_{12}}{\partial \delta}\right)^{2}\right) \frac{\partial^{3} \psi^{(+)}}{\partial \delta \partial x_{i} \partial x_{j}} \\
& \frac{\partial^{5} \psi^{(+)}}{\partial x_{i} \partial x_{j} \partial \delta^{3}}=-\frac{1}{\Pi_{12}^{2}}+4\left(\Pi_{12} \frac{\partial^{2} \Pi_{12}}{\partial \delta \partial x_{j}}+\frac{\partial \Pi_{12}}{\partial \delta} \frac{\partial \Pi_{12}}{\partial x_{j}}\right) \frac{\partial^{3} \psi^{(+)}}{\partial \delta^{2} \partial x_{i}} \\
& +2\left(\Pi_{12} \frac{\partial^{3} \Pi_{12}}{\partial \delta^{2} \partial x_{j}}+2 \frac{\partial \Pi_{12}}{\partial \delta} \frac{\partial^{2} \Pi_{12}}{\partial \delta \partial x_{j}}+\frac{\partial \Pi_{12}}{\partial x_{j}} \frac{\partial^{2} \Pi_{12}}{\partial \delta^{2}}\right) \frac{\partial^{2} \psi^{(+)}}{\partial \delta \partial x_{i}} \\
& +4 \Pi_{12} \frac{\partial \Pi_{12}}{\partial \delta} \frac{\partial^{4} \psi^{(+)}}{\partial \delta^{2} \partial x_{i} \partial x_{j}}+2 \Pi_{12} \frac{\partial \Pi_{12}}{\partial x_{j}} \frac{\partial^{4} \psi^{(+)}}{\partial \delta^{3} \partial x_{i}} \\
& \left(+6\left(\Pi_{12} \frac{\partial^{2} \Pi_{12}}{\partial \delta^{2}}+\left(\frac{\partial \Pi_{12}}{\partial \delta}\right)^{2}\right) \frac{\partial^{4} \psi^{(+)}}{\partial \delta^{2} \partial x_{i} \partial x_{j}}\right. \\
& +6 \frac{\partial \Pi_{12}}{\partial \delta} \frac{\partial^{2} \Pi_{12}}{\partial \delta^{2}} \frac{\partial^{3} \psi^{(+)}}{\partial \delta \partial x_{i} \partial x_{j}} \\
& \frac{\partial^{6} \psi^{(+)}}{\partial x_{i} \partial x_{j} \partial \delta^{4}}=-\frac{1}{\Pi_{12}^{2}} \mid \begin{array}{l}
+6\left(\Pi_{12} \frac{\partial^{2} \Pi_{12}}{\partial \delta \partial x_{j}}+\frac{\partial \Pi_{12}}{\partial \delta} \frac{\partial \Pi_{12}}{\partial x_{j}}\right) \frac{\partial^{4} \psi^{(+)}}{\partial \delta^{3} \partial x_{i}} \\
+6\left(\Pi_{12} \frac{\partial^{3} \Pi_{12}}{\partial \delta^{2} \partial x_{j}}+2 \frac{\partial \Pi_{12}}{\partial \delta} \frac{\partial^{2} \Pi_{12}}{\partial \delta \partial x_{j}}+\frac{\partial \Pi_{12}}{\partial x_{j}} \frac{\partial^{2} \Pi_{12}}{\partial \delta^{2}}\right) \frac{\partial^{3} \psi^{(+)}}{\partial \delta^{2} \partial x_{i}}
\end{array} \\
& +6\left(\frac{\partial \Pi_{12}}{\partial \delta} \frac{\partial^{3} \Pi_{12}}{\partial \delta^{2} \partial x_{j}}+\frac{\partial^{2} \Pi_{12}}{\partial \delta^{2}} \frac{\partial^{2} \Pi_{12}}{\partial \delta \partial x_{j}}\right) \frac{\partial^{2} \psi^{(+)}}{\partial \delta \partial x_{i}} \\
& +6 \Pi_{12} \frac{\partial \Pi_{12}}{\partial \delta} \frac{\partial^{5} \psi^{(+)}}{\partial \delta^{3} \partial x_{i} \partial x_{j}}+2 \Pi_{12} \frac{\partial \Pi_{12}}{\partial x_{j}} \frac{\partial^{5} \psi^{(+)}}{\partial \delta^{4} \partial x_{i}} \\
& \frac{\partial^{4} \psi^{(+)}}{\partial x_{i} \partial x_{j} \partial x_{k} \partial \delta}=-\frac{1}{\Pi_{12}^{2}}\left(\begin{array}{l}
\rho_{r} \frac{\partial^{3} \Pi_{12}}{\partial x_{i} \partial x_{j} \partial x_{k}} \\
+2\left(\Pi_{12} \frac{\partial^{2} \Pi_{12}}{\partial x_{j} \partial x_{k}}+\frac{\partial \Pi_{12}}{\partial x_{j}} \frac{\partial \Pi_{12}}{\partial x_{k}}\right) \frac{\partial^{2} \psi^{(+)}}{\partial \delta \partial x_{i}} \\
+2 \Pi_{12} \frac{\partial \Pi_{12}}{\partial x_{j}} \frac{\partial^{3} \psi^{(+)}}{\partial \delta \partial x_{i} \partial x_{k}}+2 \Pi_{12} \frac{\partial \Pi_{12}}{\partial x_{k}} \frac{\partial^{3} \psi^{(+)}}{\partial \delta \partial x_{i} \partial x_{j}}
\end{array}\right) .
\end{aligned}
$$

\subsection{Composition Derivatives of $\tau a_{\mathrm{m}}(\tau)$}

Composition derivatives of the product $\tau a_{\mathrm{m}}$ yield forms similar to Eq. (32). The first two composition derivatives are given by

$$
\frac{\partial}{\partial x_{i}}\left(\left.\frac{\partial^{n}\left[\tau a_{\mathrm{m}}(\tau)\right]}{\partial \tau^{n}}\right|_{\bar{x}}\right)=\tau \frac{\partial^{n+1}\left[a_{\mathrm{m}}(\tau)\right]}{\partial \tau^{n} \partial x_{i}}+n \frac{\partial^{n}\left[a_{\mathrm{m}}(\tau)\right]}{\partial \tau^{n-1} \partial x_{i}}
$$




$$
\frac{\partial^{2}}{\partial x_{i} \partial x_{j}}\left(\left.\frac{\partial^{n}\left[\tau a_{\mathrm{m}}(\tau)\right]}{\partial \tau^{n}}\right|_{\bar{x}}\right)=\tau \frac{\partial^{n+2}\left[a_{\mathrm{m}}(\tau)\right]}{\partial \tau^{n} \partial x_{i} \partial x_{j}}+n \frac{\partial^{n+1}\left[a_{\mathrm{m}}(\tau)\right]}{\partial \tau^{n-1} \partial x_{i} \partial x_{j}}
$$

\subsection{Composition Derivatives of $a_{\mathrm{m}}$ and $b_{\mathrm{m}}$}

As described above in Sec. 2.3, the mole fractions can be considered to either all be independent variables, or the $N$-th mole fraction can be determined based on the other $N-1$ mole fractions. The derivatives of $a_{\mathrm{m}}$ and $b_{\mathrm{m}}$ with respect to composition for both composition models are described in the sections that follow, where the composition derivatives of $a_{\mathrm{m}}$ are taken at a constant value of $\tau$, which involves the contributions from $a_{i i}$. The mixed $\tau$ and composition derivatives required in Eqs. (123) and (124) can be obtained by substituting $\frac{\partial^{n} a_{i}}{\partial \tau^{n}}$ in the place of $a_{i i}$ as required. For more information on these derivatives, see the C++ code in the supplemental information.

\subsection{1 $x_{N}$ Independent}

The summation for $a_{\mathrm{m}}$ given by Eq. (10) can also be reconstituted as two summations, one for the main diagonal (where $i=j$ ), and another for all the off-diagonal entries, which due to symmetry $\left(a_{i j}=a_{j i}\right)$ contribute two identical contributions. This results in the formulation for $a_{\mathrm{m}}$ given by

$$
a_{\mathrm{m}}=\sum_{i=1}^{N} x_{i}^{2} a_{i i}+2 \sum_{i=1}^{N-1} \sum_{j=i+1}^{N} x_{i} x_{j} a_{i j} .
$$

The first composition derivative of $a_{\mathrm{m}}$ with respect to composition when all $N$ components of the mixture are assumed to be independent is given by

$$
\left(\frac{\partial a_{\mathrm{m}}}{\partial x_{i}}\right)_{x_{j}, j \neq i}=2 \sum_{j=1}^{N} x_{j} a_{i j}
$$

The summation form of this derivative can be demonstrated for a ternary (three-component) mixture, where the pattern becomes evident, as can be seen from

$$
\begin{gathered}
a_{\mathrm{m}}=x_{1}^{2} a_{11}+x_{2}^{2} a_{22}+x_{3}^{2} a_{33}+2 x_{1} x_{2} a_{12}+2 x_{1} x_{3} a_{13}+2 x_{2} x_{3} a_{23} \\
\left(\frac{\partial a_{\mathrm{m}}}{\partial x_{1}}\right)_{x_{2}, x_{3}}=2 x_{1} a_{11}+2 x_{2} a_{12}+2 x_{3} a_{13} \\
\left(\frac{\partial a_{\mathrm{m}}}{\partial x_{2}}\right)_{x_{1}, x_{3}}=2 x_{1} a_{12}+2 x_{2} a_{22}+2 x_{3} a_{23} \\
\left(\frac{\partial a_{\mathrm{m}}}{\partial x_{3}}\right)_{x_{1}, x_{2}}=2 x_{1} a_{13}+2 x_{2} a_{23}+2 x_{3} a_{33} .
\end{gathered}
$$

The second composition derivative of $a_{\mathrm{m}}$ (for $x_{N}$ independent) is given by

$$
\left(\frac{\partial a_{\mathrm{m}}}{\partial x_{i} \partial x_{j}}\right)_{x_{k}}=2 a_{i j}
$$

for all $i$ and $j$. This result can be seen by inspection of composition derivatives of Eqs. (128), (129), and (130) for the ternary system. All further composition derivatives of $a_{\mathrm{m}}$ are equal to zero. 
The first composition derivative of $b_{\mathrm{m}}$ with respect to composition for $x_{N}$ independent and $b_{\mathrm{m}}$ with the use of linear mixing is given by

$$
\left(\frac{\partial b_{\mathrm{m}}}{\partial x_{i}}\right)_{x_{j}}=b_{i i}
$$

All further composition derivatives of $b_{\mathrm{m}}$, as well as all derivatives with respect to $\tau$, are equal to zero.

\subsection{2 $x_{N}$ Dependent}

The first $N-1$ components are independent variables, and the last component $x_{N}$ is the remainder of the mole fraction. The formula for $a_{\mathrm{m}}$ from Eq. (10) can be expressed in four pieces, one as the primary matrix for the first $(N-1) \times(N-1)$ components, one for the $N, N$ element, and two pieces for the remainder of the $N$-th row and the $N$-th column. Figure 1 shows the bands in the matrix in a graphical sense. The cells with no color correspond to the entries where both $i$ and $j$ are independent mole fractions, in red, both of the $i$ and $j$ mole fractions are dependent variables, and in green, one of the $i$ and $j$ mole fractions are dependent variables.

\begin{tabular}{|l|l|l|l|l|}
\hline $\mathrm{a}_{11}$ & $\mathrm{a}_{12}$ & $\mathrm{a}_{13}$ & $\mathrm{a}_{14}$ & $\mathrm{a}_{15}$ \\
\hline $\mathrm{a}_{21}$ & $\mathrm{a}_{22}$ & $\mathrm{a}_{23}$ & $\mathrm{a}_{24}$ & $\mathrm{a}_{25}$ \\
\hline $\mathrm{a}_{31}$ & $\mathrm{a}_{32}$ & $\mathrm{a}_{33}$ & $\mathrm{a}_{34}$ & $\mathrm{a}_{35}$ \\
\hline $\mathrm{a}_{41}$ & $\mathrm{a}_{42}$ & $\mathrm{a}_{43}$ & $\mathrm{a}_{44}$ & $\mathrm{a}_{45}$ \\
\hline $\mathrm{a}_{51}$ & $\mathrm{a}_{52}$ & $\mathrm{a}_{53}$ & $\mathrm{a}_{54}$ & $\mathrm{a}_{55}$ \\
\hline
\end{tabular}

Fig. 1. Grid of entries in $a_{m}$ for the case where $x_{N}$ is a dependent variable.

Then considering the bands in Figure 1, we can express Eq. (10) in the form

$$
a_{\mathrm{m}}=\left(\begin{array}{l}
\sum_{n=1}^{N-1} \sum_{m=1}^{N-1} x_{n} x_{m} a_{n m} \\
+\sum_{k=1}^{N-1} x_{N} x_{k} a_{N k}+\sum_{k=1}^{N-1} x_{k} x_{N} a_{k N} \\
+x_{N}^{2} a_{N N}
\end{array}\right) .
$$

The first composition derivative of $x_{N}^{2} a_{N N}$ is given by

$$
\frac{\partial\left(x_{N}^{2} a_{N N}\right)}{\partial x_{i}}=-2 x_{N} a_{N N}
$$

because $\mathrm{d} x_{N} / \mathrm{d} x_{i}=-1$. The remainder of the $N$-th row and $N$-th column can be treated in a similar fashion

$$
\frac{\partial}{\partial x_{i}}\left(\sum_{k=1}^{N-1} x_{N} x_{k} a_{N k}\right)=\sum_{k=1}^{N-1}\left(\frac{\mathrm{d} x_{N}}{\mathrm{~d} x_{i}} x_{k}+x_{N} \frac{\mathrm{d} x_{k}}{\mathrm{~d} x_{i}}\right) a_{N k},
$$


or

$$
\frac{\partial}{\partial x_{i}}\left(\sum_{k=1}^{N-1} x_{N} x_{k} a_{N k}\right)=x_{N} a_{N i}-\sum_{k=1}^{N-1} x_{k} a_{N k}
$$

because

$$
\frac{\partial x_{k}}{\partial x_{i}}= \begin{cases}1 & i=k \\ 0 & i \neq k\end{cases}
$$

which is also sometimes expressed as the Kronecker delta. The other part of the boundary to the matrix yields a similar form, given by

$$
\frac{\partial}{\partial x_{i}}\left(\sum_{k=1}^{N-1} x_{k} x_{N} a_{k N}\right)=x_{N} a_{i N}-\sum_{k=1}^{N-1} x_{k} a_{k N} .
$$

The derivative of the remaining part of $a_{\mathrm{m}}$ can be given by

$$
\frac{\partial}{\partial x_{i}}\left(\sum_{n=1}^{N-1} \sum_{m=1}^{N-1} x_{n} x_{m} a_{n m}\right)=\sum_{n=1}^{N-1} \sum_{m=1}^{N-1} a_{n m}\left(\frac{\partial x_{n}}{\partial x_{i}} x_{m}+x_{n} \frac{\partial x_{m}}{\partial x_{i}}\right)
$$

or

$$
\frac{\partial}{\partial x_{i}}\left(\sum_{n=1}^{N-1} \sum_{m=1}^{N-1} x_{n} x_{m} a_{n m}\right)=\sum_{m=1}^{N-1} x_{m} a_{i m}+\sum_{n=1}^{N-1} x_{n} a_{n i},
$$

which can ultimately all be joined together to yield

$$
\frac{\partial a_{\mathrm{m}}}{\partial x_{i}}=-2 x_{N} a_{N N}+x_{N}\left(a_{N i}+a_{i N}\right)+\sum_{k=1}^{N-1} x_{k}\left(a_{i k}+a_{k i}-a_{k N}-a_{N k}\right),
$$

which by symmetry can be simplified to

$$
\frac{\partial a_{\mathrm{m}}}{\partial x_{i}}=2\left[x_{N}\left(a_{N i}-a_{N N}\right)+\sum_{k=1}^{N-1}\left[x_{k}\left(a_{i k}-a_{k N}\right)\right]\right] .
$$

The second composition derivative (by the argument of Eq. (137)) yields

$$
\frac{\partial^{2} a_{\mathrm{m}}}{\partial x_{i} \partial x_{j}}=2\left(a_{i j}-a_{j N}-a_{N i}+a_{N N}\right),
$$

and all further composition derivatives are equal to zero.

The formula for $b_{\mathrm{m}}$ can be expressed as

$$
b_{\mathrm{m}}=\sum_{i=1}^{N-1} x_{i} b_{i i}+\left(1-\sum_{i=1}^{N-1} x_{i}\right) b_{N N},
$$

which results in

$$
b_{\mathrm{m}}=b_{N N}+\sum_{i=1}^{N-1} x_{i}\left(b_{i i}-b_{N N}\right),
$$

and therefore the first composition derivative with respect to composition for $x_{N}$ dependent is given by

$$
\left(\frac{\partial b_{\mathrm{m}}}{\partial x_{i}}\right)_{x_{j}}=b_{i i}-b_{N N} .
$$

All further composition derivatives of $b_{\mathrm{m}}$ are equal to zero. 


\section{Validation and Results}

The analytic derivatives presented here were obtained through extensive use of the open-source python symbolic math package sympy as shown in the Jupyter notebook (formerly known as IPython notebook [27]) provided as supplemental information. A few minor manual simplifications of the resulting equations were made in order to yield slightly more compact forms.

In order to assist the user in the implementation of the derivatives presented here, numerical values are tabulated in the supplemental information for the required derivatives. These derivatives cover all the partial derivatives of $\alpha^{\mathrm{r}}$ with respect to composition, $\tau, \delta$, and mixed partial derivatives thereof.

\subsection{Numerical Derivatives Not Involving Composition Derivatives}

As the analytic derivatives themselves are quite complex, it is necessary to ensure that they have been implemented properly. The most reliable way of doing this is to compare the calculated values from the numerical derivatives with the values calculated from the analytic derivatives. Here we present a small explanation of how to carry out the numerical derivatives, which mirrors the analysis presented in the supplemental information.

The numerical $\tau$ and/or $\delta$ derivatives (not including composition derivatives) are relatively straightforward. For instance, for an arbitrary term $\Lambda=f(\tau, \delta, \bar{x})$, the first $\tau$ derivative of $\Lambda$ with a second-order truncation error centered difference can be obtained from

$$
\frac{\partial \Lambda}{\partial \tau} \approx \frac{\Lambda(\tau+\Delta \tau, \delta, \bar{x})-\Lambda(\tau-\Delta \tau, \delta, \bar{x})}{2 \Delta \tau}+\mathcal{O}\left((\Delta \tau)^{2}\right),
$$

where the term $\mathcal{O}$ represents the order of the truncation error. Similarly, the first numerical partial derivative with respect to $\delta$ (and all other variables constant) with a second-order truncation error centered finite difference would be given by

$$
\frac{\partial \Lambda}{\partial \delta} \approx \frac{\Lambda(\tau, \delta+\Delta \delta, \bar{x})-\Lambda(\tau, \delta-\Delta \delta, \bar{x})}{2 \Delta \delta}+\mathcal{O}\left((\Delta \delta)^{2}\right) .
$$

The term $\Lambda$ could be a derivative term (potentially also including composition derivatives).

In some cases, first partial derivatives with higher order truncation error are needed to reduce the error in the numerical approximation to the analytic derivative. The first derivative with respect to $\delta$ with a fourth-order truncation error centered finite difference would be given by

$$
\frac{\partial \Lambda}{\partial \delta} \approx \frac{-\Lambda(\tau, \delta+2 \Delta \delta, \bar{x})+8 \Lambda(\tau, \delta+\Delta \delta, \bar{x})-8 \Lambda(\tau, \delta-\Delta \delta, \bar{x})+\Lambda(\tau, \delta-2 \Delta \delta, \bar{x})}{12 \Delta \delta}+\mathcal{O}\left((\Delta \delta)^{4}\right) .
$$

\subsection{Numerical Derivatives with Respect to Composition}

As in the above section, we consider an arbitrary function $\Lambda=f(\tau, \delta, \bar{x})$. The derivatives with respect to composition are slightly more complex because they now involve the two possibilities of $x_{N}$ being an independent variable or being dependent on the preceding $N-1$ components of the mixture. In order to carry out derivatives of $\Lambda$ with respect to one of the first $N-1$ mole fractions, we create new composition vectors with the relevant mole fraction shifted. If $x_{N}$ is an independent variable, these new composition vectors can be expressed as

$$
\begin{aligned}
\bar{x}^{(+, i)} & =\left[x_{1}, x_{2}, \ldots, x_{i}+\Delta x, \ldots, x_{N}\right] \\
\bar{x}^{(2+, i)} & =\left[x_{1}, x_{2}, \ldots, x_{i}+2 \Delta x, \ldots, x_{N}\right]
\end{aligned}
$$




$$
\begin{aligned}
\bar{x}^{(-, i)} & =\left[x_{1}, x_{2}, \ldots, x_{i}-\Delta x, \ldots, x_{N}\right] \\
\bar{x}^{(2-, i)} & =\left[x_{1}, x_{2}, \ldots, x_{i}-2 \Delta x, \ldots, x_{N}\right] .
\end{aligned}
$$

because the only composition that must be shifted is the composition of interest. With $x_{N}$ an independent variable, the sum of $\bar{x}$ will not equal one for the shifted mole fraction vectors, $\bar{x}^{(2-, i)}, \bar{x}^{(-, i)}, \bar{x}^{(+, i)}$, and $\bar{x}^{(2+, i)}$.

If on the other hand $x_{N}$ is dependent on the first $N-1$ components, the shifted composition vectors are constructed by shifting the composition of interest, as well as applying the opposite shift to the $x_{N}$ composition, as given by

$$
\begin{gathered}
\bar{x}^{(+, i)}=\left[x_{1}, x_{2}, \ldots, x_{i}+\Delta x, \ldots, x_{N}-\Delta x\right] \\
\bar{x}^{(2+, i)}=\left[x_{1}, x_{2}, \ldots, x_{i}+2 \Delta x, \ldots, x_{N}-2 \Delta x\right] \\
\bar{x}^{(-, i)}=\left[x_{1}, x_{2}, \ldots, x_{i}-\Delta x, \ldots, x_{N}+\Delta x\right] \\
\bar{x}^{(2-, i)}=\left[x_{1}, x_{2}, \ldots, x_{i}-2 \Delta x, \ldots, x_{N}+2 \Delta x\right] .
\end{gathered}
$$

The first composition partial derivative through the use of a fourth-order truncation error centered finite difference can then be expressed as

$$
\frac{\partial \Lambda}{\partial x_{i}} \approx \frac{-\Lambda\left(\tau, \delta, \bar{x}^{(2+, i)}\right)+8 \Lambda\left(\tau, \delta, \bar{x}^{(+, i)}\right)-8 \Lambda\left(\tau, \delta, \bar{x}^{(-, i)}\right)+\Lambda\left(\tau, \delta, \bar{x}^{(2-, i)}\right)}{12 \Delta x}+\mathcal{O}\left((\Delta x)^{4}\right) .
$$

This and other finite difference forms are covered in the work of Chapra and Canale [28]. Similarly, if a second-order truncation partial derivative were desired, the form of Eq. (147) could be used.

\section{Conclusions}

In this work, a generalized derivation to transform cubic equations of state to Helmholtz-explicit formulations for use in one-fluid and multi-fluid models is presented. These transformations can be used in state-of-the-art thermophysical property libraries, either to replace a fluid in the multi-fluid model, or to use the cubic equation of state in a standalone fashion to replace the mixture (or pure-fluid) model entirely. Additional validation data and a $\mathrm{C}++$ implementation of these derivatives are provided as supplemental information.

The derivatives presented here can be extended to higher orders in composition, $\tau$, or $\delta$ by continuing the symbolic mathematics analysis that is included in the supplemental information. The higherorder composition derivatives become significantly more complex, but additional derivatives with respect to $\tau$ or $\delta$ require relatively little additional work.

Aavatsmark et al. [29] developed a new cubic equation of state for carbon dioxide for the purpose of modeling carbon capture and sequestration. Their work was published while this work was underway. Fortuitously, the equation of state proposed by Aavatsmark can be readily handled with the framework proposed here, but cannot be used in tools that are based on the standard cubic equations of state (PR, SRK, etc.), providing additional motivation for the work carried out here. In their work, several sets of the parameters $\Delta_{1}, \Delta_{2}, m_{i i}, a_{0, i i}$, and $b_{i i}$ were obtained to yield the best density predictions over a few different domains. 


\section{Supplemental Materials}

- $\quad \mathrm{C}^{++}$code implementing all the analyses presented here. In the case of an inconsistency between the analytic derivatives as typeset here and the $\mathrm{C}++$ code, the $\mathrm{C}++$ code should be used because it has been numerically validated.

- Jupyter notebook demonstrating use of sympy symbolic math package (and a PDF translation of the notebook).

- $\quad$ Sample derivative data for a three-component mixture.

\section{Acknowledgments}

The authors thank the following colleagues who played important roles in the past and present development of this work: Eric Lemmon of the National Institute of Standards and Technology, who generously shared his time to discuss numerous mathematical challenges; Monika Thol and Stefan Herrig of the Ruhr-Universität Bochum, Germany, for the motivation of this work; Vladimir Diky and Diego Ortiz-Vega, who laid some of the groundwork for this analysis; Lars Hüttermann and Pit Podleschny, who carried out preliminary work on specialized derivations for PR and SRK at the Ruhr-Universität Bochum; the National Research Council for their generous support of Ian Bell's postdoctoral fellowship.

\section{References}

[1] Callen HB (1960) Thermodynamics : an introduction to the physical theories of equilibrium thermostatics and irreversible thermodynamics (Wiley, New York).

[2] Lemmon EW, Huber ML, McLinden MO (2013), NIST Standard Reference Database 23: Reference Fluid Thermodynamic and Transport Properties-REFPROP, Version 9.1, National Institute of Standards and Technology. http://www.nist.gov/srd/nist23.cfm

[3] Bell IH, Wronski J, Quoilin S, Lemort V (2014) Pure and Pseudo-pure Fluid Thermophysical Property Eval-uation and the Open-Source Thermophysical Property Library CoolProp. Ind Eng Chem Res 53(6):2498-2508. http://dx.doi.org/10.1021/ie4033999

[4] Span R, Eckermann T, Herrig S, Hielscher S, Jäger A, Thol M (2015), TREND. Thermodynamic Reference and Engineering Data 2.0. http://www.thermo.ruhr-uni-bochum.de/

[5] Span R (2000) Multiparameter Equations of State - An Accurate Source of Thermodynamic Property Data (Springer).

[6] Lemmon E, Jacobsen RT, Penoncello SG, Friend D (2000) Thermodynamic Properties of Air and Mixtures of Nitrogen, Argon, and Oxygen from 60 to $2000 \mathrm{~K}$ at Pressures to 2000 MPa. J Phys Chem Ref Data 29(3):331-385. http://dx.doi.org/10.1063/1.1285884

[7] Lemmon EW, McLinden MO, Wagner W (2009) Thermodynamic Properties of Propane. III. A Reference Equation of State for Temperatures from the Melting Line to $650 \mathrm{~K}$ and Pressures up to $1000 \mathrm{MPa}$. J Chem Eng Data 54(12):3141-3180. http://dx.doi.org/10.1021/je900217v

[8] Lemmon EW, Tillner-Roth R (1999) A Helmholtz energy equation of state for calculating the thermodynamic properties of fluid mixtures. Fluid Phase Equilib 165(1):1-21. http://dx.doi.org/10.1016/S0378-3812(99)00262-9

[9] Lemmon EW, Jacobsen RT (1999) A Generalized Model for the Thermodynamic Properties of Mixtures. Int J Thermophys 20(3):825-835. http://dx.doi.org/10.1023/A:1022627001338

[10] Lemmon EW (1996) A Generalized Model for the Prediction of the Thermodynamic Properties of Mixtures Including VaporLiquid Equilibrium, Ph.D. thesis, University of Idaho, Moscow.

[11] Kunz O, Klimeck R, Wagner W, Jaeschke M (2007) The GERG-2004 Wide-Range Equation of State for Natural Gases and Other Mixtures (VDI Verlag GmbH).

[12] Kunz O, Wagner W (2012) The GERG-2008 Wide-Range Equation of State for Natural Gases and Other Mixtures: An Expansion of GERG-2004. J Chem Eng Data 57(11):3032-3091. http://dx.doi.org/10.1021/je300655b

[13] Gernert J, Jäger A, Span R (2014) Calculation of phase equilibria for multi-component mixtures using highly accurate Helmholtz energy equations of state. Fluid Phase Equilibr 375:209-218. http://dx.doi.org/10.1016/j.fluid.2014.05.012

[14] Gernert J, Span R (2016) EOS-CG: A Helmholtz energy mixture model for humid gases and CCS mixtures. J Chem Thermodyn 93:274-293. http://dx.doi.org/10.1016/j.jct.2015.05.015

[15] Soave G (1972) Equilibrium Constants from a Modified Redlich-Kwong Equation of State. Chem Eng Sci 27(6):1197-1203. http://dx.doi.org/10.1016/0009-2509(72)80096-4

[16] Redlich O, Kwong JN (1949) On the thermodynamics of solutions. V. An equation of state. Fugacities of gaseous solutions. Chem Rev 44(1):233-244. http://dx.doi.org/10.1021/Cr60137a013 
[17] Peng DY, Robinson DB (1976) A new two-constant equation of state. Ind Eng Chem Fundamen 15(1):59- 64. http://dx.doi.org/10.1021/i160057a011

[18] Michelsen ML, Mollerup JM (2007) Thermodynamic Models: Fundamentals \& Computational Aspects (Tie-Line Publications).

[19] Poling BE, Prausnitz JM, O’Connell JP (2001) The Properties of Gases and Liquids 5th edition (McGraw Hill).

[20] Wei YS, Sadus RJ (2000) Equations of State for the Calculation of Fluid-Phase Equilibria. AICHE J 46(1):169-196. http://dx.doi.org/10.1002/aic.690460119

[21] Valderrama J (2003) The State of the Cubic Equations of State. Ind Eng Chem Res 42(8):1603-1618. http://dx.doi.org/10.1021/ie020447b

[22] van der Waals JD (1873) Over de Continuiteit van den Gas- en Vloeistoftoestand, Ph.D. thesis, University of Leiden.

[23] Robinson DB, Peng DY (1978) The characterization of the heptanes and heavier fractions for the GPA Peng-Robinson programs (Research Report RR-28) (Gas Processors Association).

[24] Mathias PM, Copeman TW (1983) Extension of the Peng-Robinson equation of state to complex mixtures: evaluation of the various forms of the local composition concept. Fluid Phase Equilibr 13:91-108. http://dx.doi.org/10.1016/03783812(83)80084-3

[25] Horstmann S, Jabloniec A, Krafczyk J, Fischer K, Gmehling J (2005) PSRK group contribution equation of state: comprehensive revision and extension IV, including critical constants and $\alpha$-function parameters for 1000 components. Fluid Phase Equilibr 227(2):157-164. http://dx.doi.org/10.1016/j.fluid.2004.11.002

[26] McFarlane RA, Gray MR, Shaw JM (2010) Evaluation of co-volume mixing rules for bitumen liquid density and bubble pressure estimation. Fluid Phase Equilibr 293(1):87-100. http://dx.doi.org/10.1016/j.fluid.2010.02.032

[27] Perez F, Granger BE (2007) IPython: a system for interactive scientific computing. Comput Sci Eng 9(3):21-29. http://dx.doi.org/10.1109/Mcse.2007.53

[28] Chapra SC, Canale RP (2015) Numerical Methods for Engineers 7th edition (McGraw Hill)

[29] Aavatsmark I, Kometa BK, Gasda SE, Sandve TH, Nilsen HM (2015) A Generalized Cubic Equation of State with Application to Pure CO2 Injection in Aquifers. Computational Geosciences 20(3):623-635. http://dx.doi.org/10.1007/s10596-015-9537-0

About the authors: Ian Bell is a National Research Council postdoctoral researcher in the Applied Chemicals and Materials Division of the Materials and Measurements Laboratory of NIST. He conducts research in the modeling of the thermophysical properties of pure fluids and mixtures. Andreas Jäger is a research assistant at the Technische Universität Dresden (Dresden University of Technology) in Germany. His primary research focuses on equations of state for fluid and solid phases and phase equilibrium calculations. The National Institute of Standards and Technology is an agency of the U.S. Department of Commerce. 